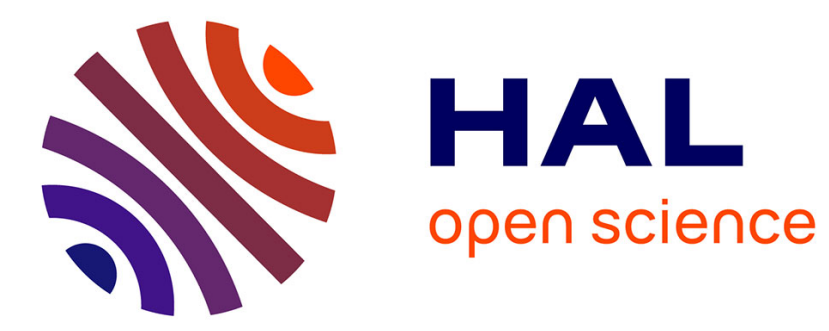

\title{
New ferrocenyl P,O ligands with polar substituents
} Catherine Audin, Jean-Claude Daran, Éric Deydier, Eric Manoury, Rinaldo Poli

\section{To cite this version:}

Catherine Audin, Jean-Claude Daran, Éric Deydier, Eric Manoury, Rinaldo Poli. New ferrocenyl P,O ligands with polar substituents. Comptes Rendus. Chimie, 2010, 13 (8-9), pp.890-899. 10.1016/j.crci.2010.05.005 . hal-03177735

\section{HAL Id: hal-03177735 \\ https://hal.science/hal-03177735}

Submitted on 23 Mar 2021

HAL is a multi-disciplinary open access archive for the deposit and dissemination of scientific research documents, whether they are published or not. The documents may come from teaching and research institutions in France or abroad, or from public or private research centers.
L'archive ouverte pluridisciplinaire HAL, est destinée au dépôt et à la diffusion de documents scientifiques de niveau recherche, publiés ou non, émanant des établissements d'enseignement et de recherche français ou étrangers, des laboratoires publics ou privés. 


\title{
New ferrocenyl P,O ligands with polar substituents
}

\author{
Cathérine Audin, ${ }^{1,2}$ Jean-Claude Daran, ${ }^{2}$ Eric Deydier*, ${ }^{1,2}$ Eric Manoury*2 and \\ Rinaldo Poli 2,3
}

1 Université de Toulouse; UPS, Institut Universitaire de Technologie Paul Sabatier; Département de Chimie; Av. Georges Pompidou, BP 20258, F-81104 Castres Cedex, France Fax: (+) 33-563356388; E-mail:

eric.deydier@iut-tlse3.fr

2 CNRS; LCC (Laboratoire de Chimie de Coordination); Université de Toulouse; UPS, INP; F-31077 Toulouse,

France ; 205, route de Narbonne, F-31077 Toulouse, France; Fax: (+) 33-561553003; E-mail :

eric.manoury@lcc-toulouse.fr

3 Institut Universitaire de France, 103, bd Saint-Michel, 75005 Paris, France

\begin{abstract}
A new ferrocenyl P,O ligand has been synthesized and successfully used in the SuzukiMiyaura coupling reaction of phenylboronic acid with bromobenzene. Some analogues carrying charged (imidazolium) or neutral (monomethylether PEG 750, tetraethylbisphosphonate) polar substituents have been prepared by functionalization of a common synthetic intermediate easily obtained using bromoalcohol $p-\mathrm{HOCH}_{2}\left(\mathrm{C}_{6} \mathrm{H}_{4}\right) \mathrm{CH}_{2} \mathrm{Br}$ as bifunctional linker.
\end{abstract}

Keywords: Ferrocene, Phosphine, PEG, Bisphosphonate, Imidazolium, Catalysis, Suzuki coupling.

\section{Résumé}

Un nouveau ligand $\mathrm{P}, \mathrm{O}$ ferrocénique a été synthétisé et utilisé avec succès dans la réaction de couplage de Suzuki entre l'acide phénylboronique et le bromobenzène. Des analogues portant des substituants polaires chargés (imidazolium) ou neutres (monométhyléther du PEG 750, tétraéthylbisphosphonate) ont été préparés à partir d'un intermédiaire synthétique commun, aisément obtenu en utilisant le bromoalcool $p-\mathrm{HOCH}_{2}\left(\mathrm{C}_{6} \mathrm{H}_{4}\right) \mathrm{CH}_{2} \mathrm{Br}$ comme lien bifonctionnel.

Mots-clés: Ferrocène, Phosphine, PEG, Bisphosphonate, Imidazolium, Catalyse, Couplage de Suzuki.

\footnotetext{
* Corresponding authors. fax: +33 5615530 03; e-mail address: eric.deydier@iut-tlse3.fr, and manoury@lcc$\underline{\text { toulouse.fr. }}$
} 


\section{Introduction}

Over the last few decades, homogenous asymmetric catalysis by transition metals has received considerable attention and numerous chiral ligands and complexes allowing high efficiency reactions have been reported [1]. However, only few examples have been transferred to industrial processes [2], in many cases because of the expenses associated to catalyst loss. The efficient separation of expensive catalysts and ligands to enable reuse in subsequent cycles is a main challenge that meets both industrial economical needs and new stricter environmental regulations.

Three major strategies have been reported for immobilizing homogeneous catalyst $[3,4]$. The first one consists of immobilization on solid supports such as polymers [5,6] or inorganic materials [7] (silica, metal oxides, metal phosphates....). At the end of the reaction, the catalyst is easily recovered by filtration, but its activity and selectivity are often lower relative to the corresponding homogeneous catalyst. As a second approach, in order to combine the advantages of heterogeneous and homogeneous catalysis, soluble polymers [8] or dendrimers [9] have been used as catalyst support. The reaction occurs then in homogeneous media and the catalyst is finally recovered by precipitation or by filtration through membranes. However, longer synthetic procedures are involved because of the functionalization needed to link the catalyst to the support. The third strategy, involving non conventional liquid media and multiphasic systems with immobilization of the homogeneous catalyst on a "liquid carrier" has attracted increasing interest $[10,11]$. The functionalized catalyst, with a specifically designed ligand, is isolated in one phase at the end of the reaction, while the product remains in the other phase allowing easy product isolation and catalyst reuse. Among these "liquid carriers", supercritical carbon dioxide [12], perfluorinated solvents [13], ionic liquids [14,15], water [16,17] and polyethylene glycols (PEG) [18] have emerged as interesting candidates.

We have long been interested in the design and the synthesis of new chiral catalysts for exploring new asymmetric catalytic reactions or for improving existing ones $[19,20,21,22,23,24,25,26,27]$. In this area, chiral phosphines have played a significant role. The possibility to easily modify their electronic and steric properties by a judicious choice of their substituents has proven extremely useful to successfully optimized catalytic reactions. Among the numerous phosphine ligands reported to date, ferrocenyl phosphines functionalized by an oxygen atom (PO ferrocenyl phosphines) constitute a distinct class of hemilabile ligands attracting increasing interest [28,29,30,31,32]. We have recently developed promising PO ferrocenyl ligands in an enantiomerically pure form (planar chirality) and started investigating their efficiency in 
asymmetric catalysis (allylic substitution) [23]. In addition, we recently took interest in improving catalyst recycling using ionic liquid, water or PEG as catalyst "liquid carriers" and in investigating the influence of these media on both the catalytic reaction and the recycling efficiency. As a first part of our project, we have decided to develop simple chiral phosphine-ethers as new ligands for catalysis in unusual solvents. In this manuscript, we will describe the synthesis of the first members of this new family of phosphine-ethers, their use in Suzuki-Miyura reaction and the synthesis of new PO ferrocenyl ligands bearing charged (imidazolium) or neutral (monomethylether PEG 750, tetraethylbisphosphonate) polar tags [33] for future catalytic application in non conventional media.

\section{Results and discussion}

As an entry to new PO ferrocenyl ligands, we have chosen 2thiodiphenylphosphino(hydroxymethyl)ferrocene, 1 (Scheme 1). This compound can be prepared in multigram quantities and isolated as a racemic mixture or in a pure enantiomeric form, opening direct access to chiral ligands [23]. The phosphino function is protected from oxidation by a sulphur atom allowing working in air. Its functionalization can be performed in a one pot process by successive addition of a strong acid $\left(\mathrm{HBF}_{4}\right)$, generating probably a ferrocenyl carbocation, and then the nucleophile of choice (alcohol, thiol...) usually leading to the expected substitution product in high yields [21]. In order to obtain a large variety of tagged ligands, we needed a synthetic intermediate which bears both a PO ferrocenyl framework and a function (Y) easily transformed into R' tags (Scheme 1). For this purpose, we have designed a bifunctional linker (HO-linker-Y). The bromoalcohol $p-\mathrm{HOCH}_{2}\left(\mathrm{C}_{6} \mathrm{H}_{4}\right) \mathrm{CH}_{2} \mathrm{Br}$ appears to be an interesting first candidate to achieve this task. The benzylic bromide may be easily substituted by various R' substrates which will increase PO ferrocenyl ligand affinity for "liquids carriers" such as water, ionic liquids or PEG. Moreover, the relative para arrangement of the $\mathrm{CH}_{2}-\mathrm{OH}$ and $\mathrm{CH}_{2}-\mathrm{Br}$ groups allows the $\mathrm{R}$ ' tag to be located remotely from the ligand coordinating atoms $(\mathrm{P}, \mathrm{O})$. However, before embarking in this functionalization work, we first wished to establish the ligand efficiency in the Suzuki-Miyaura reaction with the simplest member (compound $\mathbf{2 b}$ ) of this new family of phosphine-ethers (Scheme 2).

\subsection{Synthesis of ligand $\mathbf{2 b}$}

Compound $\mathbf{2 b}$ was readily prepared in good yields by addition of an excess of paramethylbenzylalcohol $p-\mathrm{HOCH}_{2}\left(\mathrm{C}_{6} \mathrm{H}_{4}\right) \mathrm{CH}_{3}$ to racemic 1 in the presence of tetrafluoroboric acid 
in dichloromethane, according to the reaction conditions previously optimized for the synthesis of ferrocenyl phosphine thioethers [21]. After purification by flash chromatography on silicagel, compound 2a was obtained as a yellow powder in $45 \%$ yield. Desulfurization was achieved with high efficiency using tris(dimethylamino)phosphine in refluxing toluene for twelve hours under an argon atmosphere. 2b was recovered as yellow oil after flash chromatography (70\% yield). Both compounds have been fully characterized by mass spectrometry and multinuclear NMR. Selected NMR values are reported in Table 1.

\subsection{Preliminary evaluation of $P O$ ferrocenyl ligand efficiency in a Suzuki catalytic reaction.}

Ligand $\mathbf{2 b}$ was tested in the coupling reaction of phenylboronic acid with bromobenzene in the presence of $\mathrm{Pd}_{2}(\mathrm{dba})_{3}\left(1.0 \%\right.$ molar of $\mathrm{Pd}$ ) as catalyst and $\mathrm{Cs}_{2} \mathrm{CO}_{3}$ as base (see Scheme 3). A control experiment was also carried out under the same conditions but without ligand $\mathbf{2 b}$. Figure 1 reports the phenyl bromide consumption (\%) and the biphenyl formation (\%) versus time for both runs. Whereas the reaction is complete within two hours when using ligand $\mathbf{2} \mathbf{b}$ at $70^{\circ} \mathrm{C}$, the conversion remained very low (less than 10\%) for the control experiment without ligand $\mathbf{2 b}$, even after seven hours. Comparisons to recently published P,O ferrocenyl ligands underline the efficiency of ligand $\mathbf{2 b}$ in the Suzuki-Miyaura reaction [29,32]. For example, Shi et al. [32] obtained a $96 \%$ isolated yield of biphenyl in 6 hours at $110^{\circ} \mathrm{C}$ using a polymersupported PO ferrocenyl ligand ( $c f$. with a $98 \%$ conversion after only two hours at $70^{\circ} \mathrm{C}$ using 2b). Using a similar molar ratio, Hor et al. [29] reported isolated yields of $80-90 \%$ at $60^{\circ} \mathrm{C}$ in THF but only after 5 hours of reaction. These results, obtained under non optimized conditions, confirm the interest of such P,O ligands in Suzuki-Miyaura reactions.

\subsection{Synthesis of tagged ligands}

Our next goal was to develop recyclable catalytic systems using water, RT ionic liquids or PEG as "liquid carriers", in particular for Suzuki reaction which have already been efficiently carried out in such solvents [34]. Therefore, we synthesized analogues of ligand $\mathbf{2 b}$ bearing various substituents in order to increase their solubility in the "liquid carrier". All of these tagged ligands have been prepared from the same synthetic intermediate, compound $\mathbf{3}$ (Scheme $4)$.

\subsubsection{Preparation of synthetic intermediate 3}


Like 2a, compound $\mathbf{3}$ was readily prepared in good yields by addition of an excess of $p$ $\mathrm{HOCH}_{2}\left(\mathrm{C}_{6} \mathrm{H}_{4}\right) \mathrm{CH}_{2} \mathrm{Br}$ to 1 in the presence of tetrafluoroboric acid in dichloromethane. After purification by flash chromatography on silicagel, compound $\mathbf{3}$ was obtained as a yellow powder with an overall yield of $70 \%$. Its structure was confirmed by NMR, mass spectrometry and X-Ray diffraction analyses of the racemic mixture (Table 2). A view of the compound is shown in Figure 2. As observed in related ferrocenyl derivatives [20,22,23,24,26,35], the sulfur is endo with respect to the $\mathrm{Cp}$ ring, being away from the ring plane by 0.808(6) $\AA$, whereas the oxygen is exo by $1.352(5) \AA$. The two Cp rings are nearly parallel (dihedral angle of $2.6(2)^{\circ}$ ) and roughly eclipsed (twist angle of $\left.7.7(2)^{\circ}\right)$. The C2-C21-O2-C22 atoms are nearly planar with the largest deviations from the mean plane being 0.085(2) $\AA$ for $\mathrm{C} 22$ and this plane is nearly perpendicular to the phenyl ring, with which it has a dihedral angle of $89.5(1)^{\circ}$. The $\mathrm{O} 2$ and $\mathrm{Br} 1$ atoms are anti with respect to the $\mathrm{C} 22---\mathrm{C} 23$ axis. All bonds lengths and angles in this structure are within expected range (Table 3). NMR data are in agreement with the expected values. The $\mathrm{C}_{23}$ atom (bonded to bromide) gives a signal around $\delta=35 \mathrm{ppm}$ in the ${ }^{13} \mathrm{C} \mathrm{NMR}$ $\left(\mathrm{CDCl}_{3}\right)$, upfield relative to the $\mathrm{C}$ atoms of the other $\mathrm{CH}_{2}$ groups $(\delta>70)$. This signal is an efficient probe to follow the bromide substitution by the various tags. Selected values are reported in Table 1.

\subsubsection{Synthesis of tagged-ligands}

The last step of our synthetic work consists in increasing the affinity of the ligands with water, ionic liquid or PEG solvents by introducing carefully chosen R' fragments (Scheme 1). Three different polar tags have been selected to accomplish this task (Scheme 4). The ligand polarity may be increased by introducing the tetraethylbisphosphonate fragment, already used previously to synthesize water soluble phosphine [36], to generate compound $\mathbf{4 b}$. Bisphosphonate esters are also potential precursors of highly charged bisphosphonate anions, $\mathrm{R}_{2} \mathrm{C}\left(\mathrm{PO}_{3}{ }^{2-}\right)_{2}$, which may increase water solubility, although they may also compete with the PO donating atoms of the core ligand for metal coordination. Such anionic ligands could be future synthetic targets. We also imagine increasing the ligand solubility by grafting an imidazolium moiety (compound $\mathbf{5 b}$ ), a cation frequently found in ionic liquids. This cation is specifically designed for use in water or ionic liquid media. The third considered fragment is a monomethylether PEG750 (compound 6b), in order to increase the ligand solubility in water or PEG media.

Ligand $\mathbf{4 b}$ was obtained from $\mathbf{3}$ in a two step synthesis via compound $\mathbf{4 a}$ (Scheme 4). The methodology involved the reactive tetraethyl methylenebisphosphonate carbanion, 
prepared from the treatment of tetraethyl methylenebisphosphonate with sodium hydride in THF at $0^{\circ} \mathrm{C}$, which efficiently replaced the bromide ion in compound $\mathbf{3}$. The moderate yield of $40 \%$ for this step is partially explained by the subsequent formation of the dialkylated compound 4c (Scheme 5). In agreement with published results [37], this reaction gave mainly monoalkylated product $4 \mathbf{a}$ with small amount $(60 \mathrm{mg})$ of dialkylated $4 \mathbf{c}$ (12\% of the consumed 3) and unreacted starting material. The desulfurization of $\mathbf{4 a}$ was achieved with high efficiency using tris(dimethylamino)phosphine in refluxing toluene for twelve hours under argon atmosphere. $\mathbf{4 b}$ was obtained as an orange oil after flash chromatographic purification (90\% overall yield). Compounds $\mathbf{4 a}, \mathbf{4 b}, \mathbf{4 c}$ have been isolated and completely characterized by mass spectrometry and multinuclear NMR. Significant NMR signals are reported in Table 1.

The synthesis of compound 5a was successfully carried out by addition of methylimidazole on compound $\mathbf{3}$ in refluxing toluene. It was isolated by filtration as a pure yellow powder in a relatively good yield (70\%). The same desulfurization procedure used for 4b was not applied because of the high imidazolium affinity to silicagel. Therefore, we turned our attention to a procedure involving heterogeneous conditions $[38,26]$. Desulfurization was performed by reacting 5a with Raney $\mathrm{Ni}$ in acetonitrile under an argon atmosphere at $50^{\circ} \mathrm{C}$. Compound $\mathbf{5 b}$ was isolated as a yellow solid in quantitative yield after filtration. Significant NMR signals for both $\mathbf{5 a}$ and $\mathbf{5 b}$ are reported in Table 1.

The third target is a $\mathrm{P}, \mathrm{O}$ ferrocenyl ligand bearing a polyethyleneglycol chain. We have chosen PEG 750 monomethylether. ${ }^{1} \mathrm{H}$ NMR integration confirmed the general formula of this PEG: $\mathrm{MeO}\left(\mathrm{CH}_{2} \mathrm{CH}_{2} \mathrm{O}\right)_{16.3} \mathrm{H}$. Compound 6a was obtained by addition of an excess PEG 750 monomethylether alcoholate, prepared by treatment of PEG 750 monomethylether with sodium hydride, on compound 3 in $\mathrm{THF}$ at $0^{\circ} \mathrm{C}$. Compound $6 \mathrm{a}$ was isolated as a yellow wax in $60 \%$ yield. This low value compared to the crude reaction yield is due to the water solubility of $6 \mathbf{6}$ leading to partial loss during the extraction and washing to eliminate the PEG 750 excess. Desulfurization was carried out using tris(dimethylamino)phosphine (Scheme 4) followed by flash chromatographic purification, yielding compound $\mathbf{6 b}$. Compounds $\mathbf{6 a}$ and $\mathbf{6 b}$ were fully characterised by multinuclear NMR.

\subsection{Conclusion}

Chiral phosphine-ether $\mathbf{2 b}$ has been synthesized and successfully used in a Suzuki-Miyaura coupling reaction. In addition, tagged analogues of $\mathbf{2 b}$ bearing various polar substituents (bisphosphonate, imidazolium, PEG750 monomethylether groups) have been prepared from the 
same synthetic intermediate, compound $\mathbf{3}$. All of these ligands have been obtained as racemic mixtures but may be easily obtained in an enantiomerically pure form starting from the enantiomerically pure alcohol $\mathbf{1}$, allowing their use in asymmetric catalysis [39]. The use of this new P,O ligands in catalytic reaction (Suzuki reaction,..) in non-classical media and the assessment of their efficiency in catalyst recycling is currently underway.

\section{Experimental}

\subsection{General}

All reactions were carried out using conventional Schlenk techniques under an inert atmosphere of argon. Thin layer chromatography and flash chromatography were carried out on Merck Kieselgel $60 \mathrm{~F}_{254}$ precoated aluminum plates and Merck Kieselgel 60 respectively. Solvents were dried by conventional methods before use. Commercial samples were used as received without further purification (tetrafluoroboric acid diethylether complex, tetraethylmethylenebisphosphonate, $\mathrm{N}$-methylimidazole, $\mathrm{Pd}_{2}(\mathrm{dba})_{3}$, Raney ${ }^{\circledR} 2800$ slurry in $\mathrm{H}_{2} \mathrm{O}$ and phenylboronic acid from Sigma Aldrich; tris(dimethylamino)phosphine, monomethylether mPEG 750 and diethylene glycol dibutyl ether from Alfa Aesar; bromobenzene from Janssen Chimica). GC analyses were realized with an Agilent 6890 chromatograph equipped with FID detector, automatic sampler and HP5-MS capillary column (30m x $0.25 \mathrm{~mm} \times 0.25 \mu \mathrm{m})$. NMR analyses were performed on Bruker AV500, AV300 and DPX200 instruments. The spectra were referenced internally using the signal from the residual solvent for ${ }^{1} \mathrm{H}$ and ${ }^{13} \mathrm{C}$, and externally using $85 \% \mathrm{H}_{3} \mathrm{PO}_{4}$ for ${ }^{31} \mathrm{P}$. Chemical shifts and coupling constants are given in ppm and Hertz respectively. The following abbreviations are used: s, singulet; d, doublet; t, triplet; quint, quintuplet; m, multiplet; br, broad. Elemental analyses were realized by "Service d'Analyse du Laboratoire de Chimie de Coordination", Toulouse, France. Mass spectral analyses $\left(\mathrm{FAB}^{+}\right.$with MNBA matrix; HR MASS) were performed by "Service Commun de Spectrométrie de Masse" of the Université Paul Sabatier, Toulouse (France).

\subsection{Syntheses}

Racemic 2-thiodiphenylphosphino(hydroxymethyl)ferrocene, 1, was synthesized according to published procedure [40].

\subsubsection{Synthesis and characterization of compound $2 a$}


In a Schlenk tube, $0.75 \mathrm{~g}$ of $\mathbf{1}(1.74 \mathrm{mmol})$ was dissolved in $8 \mathrm{~mL}$ of dry dichloromethane. A $54 \%$ solution of tetrafluoroboric acid in ether $(0.73 \mathrm{~mL}, 5.30 \mathrm{mmol})$ was then added. After 1 min stirring, a solution of $2.5 \mathrm{~g}$ of 4-methylbenzylalcohol $(20.5 \mathrm{mmol})$ in $8 \mathrm{~mL}$ of dry dichloromethane was added. After 1 min of stirring, the crude material was filtered on silica gel with ether as eluent. After evaporation of the solvent, $0.4 \mathrm{~g}$ of $\mathbf{2 a}$ was obtained as a yellow solid (yield = 43\%). ${ }^{1} \mathrm{H}$ NMR (200.1 MHz, $\left.\mathrm{CDCl}_{3}\right), \delta(\mathrm{ppm}): 7.90-7.65$ (4H, m: $\left.\mathrm{PPh}_{2}\right) ; 7.52-$ 7.34 (6H, m: $\left.\mathrm{PPh}_{2}\right) ; 7.05$ (2H, d, J = 7.9 Hz: Ph); 6.95 (2H, d, J = 7.9 Hz: Ph); 4.89 (1H, d, J = $\left.10.9 \mathrm{~Hz}: \mathrm{CpCH}_{2}\right)$; 4.66 (1H, m: subst Cp); $4.45\left(1 \mathrm{H}, \mathrm{d}, \mathrm{J}=10.9 \mathrm{~Hz}: \mathrm{CpCH}_{2}\right) ; 4.35$ (1H, m: subst Cp); 4.33 (5H, s: Cp); 4.29 (2H, d: 2.9Hz: $\left.\mathrm{PhCH}_{2} \mathrm{O}\right) ; 3.85$ (1H, m: subst Cp); 2.33 (3H, m: $\mathrm{CH}_{3}$ ). ${ }^{13} \mathrm{C} \mathrm{NMR}\left(50.3 \mathrm{MHz}, \mathrm{CDCl}_{3}\right.$ ), $\delta(\mathrm{ppm}): 136.8$ (s: quat $\mathrm{Ph}$ ); 135.4 (s: quat $\mathrm{Ph}$ ); 134.8 $\left(\mathrm{d}, \mathrm{J}_{\mathrm{PC}}=87.1 \mathrm{~Hz}:\right.$ quat $\left.\mathrm{PPh}_{2}\right) ; 133.6\left(\mathrm{~d}, \mathrm{~J}_{\mathrm{PC}}=86.1 \mathrm{~Hz}:\right.$ quat $\left.\mathrm{PPh}_{2}\right) ; 132.2\left(\mathrm{~d}, \mathrm{~J}_{\mathrm{PC}}=10.7 \mathrm{~Hz}\right.$ : $\left.\mathrm{PPh}_{2}\right) ; 132.1\left(\mathrm{~d}, \mathrm{~J}_{\mathrm{PC}}=10.7 \mathrm{~Hz}: \mathrm{PPh}_{2}\right) ; 131.2\left(\mathrm{~d}, \mathrm{~J}_{\mathrm{PC}}=2.2 \mathrm{~Hz}: \mathrm{PPh}_{2}\right) ; 131.1\left(\mathrm{~d}, \mathrm{~J}_{\mathrm{PC}}=2.2 \mathrm{~Hz}\right.$ : $\left.\mathrm{PPh}_{2}\right) ; 128.8$ (s: Ph); 128.1 (d, JPC $\left.=12.3 \mathrm{~Hz}: \mathrm{PPh}_{2}\right) ; 128.0$ (d, JPC $\left.=12.3 \mathrm{~Hz}: \mathrm{PPh}_{2}\right) ; 127.7(\mathrm{~s}:$ $\mathrm{Ph}) ; 88.3\left(\mathrm{~d}, \mathrm{~J}_{\mathrm{PC}}=12.1 \mathrm{~Hz}\right.$ : quat $\left.\mathrm{Cp}\right) ; 75.2\left(\mathrm{~d}, \mathrm{~J}_{\mathrm{PC}}=12.5 \mathrm{~Hz}\right.$ : subst Cp); $75.6\left(\mathrm{~d}, \mathrm{~J}_{\mathrm{PC}}=94.7 \mathrm{~Hz}\right.$ : quat Cp); $74.5\left(\mathrm{~d}, \mathrm{~J}_{\mathrm{PC}}=9.4 \mathrm{~Hz}\right.$ : subst Cp); $72.4\left(\mathrm{~s}, \mathrm{PhCH}_{2} \mathrm{O}\right) ; 70.7$ (s: Cp); 69.4 (d, $\mathrm{J}_{\mathrm{PC}}=10.4$ Hz: subst Cp); 66.7 (s: $\mathrm{CpCH}_{2} \mathrm{O}$ ); 21.3 (s: $\left.\mathrm{CH}_{3}\right) .{ }^{31} \mathrm{P}$ NMR (81.0 MHz, $\mathrm{CDCl}_{3}$ ), $\delta$ (ppm): 43.1. HR MS (DCI CH 4$), \mathrm{C}_{31} \mathrm{H}_{29} \mathrm{OPSFe}$, calcd. mass [M]: 536.1026; exp. mass [M]: 536.1039.

\subsubsection{Synthesis and characterization of compound $\mathbf{2 b}$}

In a Schlenk tube, $350 \mathrm{mg}$ of $\mathbf{2 a}(0.22 \mathrm{mmol})$ were dissolved in $5 \mathrm{~mL}$ of dry toluene together with $0.75 \mathrm{~mL}$ of tris(dimethylamino)phosphine $(3.9 \mathrm{mmol})$. The solution was kept at reflux overnight under argon. After cooling back to room temperature, the solution was evaporated in уасио. The crude residue was purified under argon by flash chromatography on silica gel with dichloromethane as eluent. After evaporation of the solvent, $235 \mathrm{mg}$ of $\mathbf{2 b}$ were obtained as an orange waxy solid (yield =70\%). ${ }^{1} \mathrm{H}$ NMR $\left(200.1 \mathrm{MHz}, \mathrm{CDCl}_{3}\right), \delta(\mathrm{ppm}): 7.67-7.58(2 \mathrm{H}, \mathrm{m}$ : $\left.\mathrm{PPh}_{2}\right) ; 7.48-7.41$ (3H, m: $\left.\mathrm{PPh}_{2}\right) ; 7.32-7.29$ (5H, m: $\left.\mathrm{PPh}_{2}\right) ; 7.08$ (2H, d, J = 8.1 Hz: Ph); 6.97 $(2 \mathrm{H}, \mathrm{d}, \mathrm{J}=8.1 \mathrm{~Hz}: \mathrm{Ph}) ; 4.65\left(1 \mathrm{H}, \mathrm{dd}, \mathrm{J}=10.9 \mathrm{~Hz}, \mathrm{~J}_{\mathrm{PH}}=2.2 \mathrm{~Hz}: \mathrm{CpCH}_{2}\right) ; 4.60$ (1H, m: subst $\mathrm{Cp}) ; 4.44\left(1 \mathrm{H}, \mathrm{d}, \mathrm{J}=10.9 \mathrm{~Hz}: \mathrm{CpCH}_{2}\right) ; 4.37\left(2 \mathrm{H}, \mathrm{s}: \mathrm{PhCH}_{2} \mathrm{O}\right) ; 4.36$ (1H, m: subst Cp); 4.08 (5H, s: Cp); $3.83(1 \mathrm{H}, \mathrm{m}$ : subst $\mathrm{Cp}) ; 2.37\left(3 \mathrm{H}, \mathrm{m}: \mathrm{CH}_{3}\right) .{ }^{13} \mathrm{C} \mathrm{NMR}\left(50.3 \mathrm{MHz}, \mathrm{CDCl}_{3}\right), \delta$ (ppm): $140.8\left(\mathrm{~d}, \mathrm{~J}_{\mathrm{PC}}=9.3 \mathrm{~Hz}:\right.$ quat $\left.\mathrm{PPh}_{2}\right) ; 138.3\left(\mathrm{~d}, \mathrm{~J}_{\mathrm{PC}}=8.2 \mathrm{~Hz}:\right.$ quat $\left.\mathrm{PPh}_{2}\right) ; 137.6(\mathrm{~s}:$ quat $\mathrm{Ph}) ; 136.2$ (s: quat Ph); $135.9\left(\mathrm{~d}, \mathrm{~J}_{\mathrm{PC}}=20.3 \mathrm{~Hz}: \mathrm{PPh}_{2}\right) ; 133.2\left(\mathrm{~d}, \mathrm{~J}_{\mathrm{PC}}=17.9 \mathrm{~Hz}: \mathrm{PPh}_{2}\right) ; 129.9(\mathrm{~s}$ $\left.: \mathrm{PPh}_{2}\right) ; 129.6(\mathrm{~s}: \mathrm{Ph}) ; 128.9\left(\mathrm{~d}, \mathrm{~J}_{\mathrm{PC}}=7.5 \mathrm{~Hz}: \mathrm{PPh}_{2}\right) ; 128.8\left(\mathrm{~d}, \mathrm{~J}_{\mathrm{PC}}=5.7 \mathrm{~Hz}: \mathrm{PPh}_{2}\right) ; 128.5(\mathrm{~s}:$ $\left.\mathrm{PPh}_{2}\right) ; 128.4$ (s: $\left.\mathrm{Ph}\right) ; 90.1$ (d, $\mathrm{J}_{\mathrm{PC}}=24.6 \mathrm{~Hz}$ : quat Cp); 77.5 (d, $\mathrm{J}_{\mathrm{PC}}=8.1 \mathrm{~Hz}:$ quat Cp); 73.5 (d, 
$\mathrm{J}_{\mathrm{PC}}=3.4 \mathrm{~Hz}$ : subst $\left.\mathrm{Cp}\right) ; 72.9\left(\mathrm{~s}, \mathrm{PhCH}_{2} \mathrm{O}\right) ; 72.7$ (d, $\mathrm{J}_{\mathrm{PC}}=3.7 \mathrm{~Hz}$ : subst Cp); 70.6 (s: subst Cp); 70.3 (s: $\mathrm{Cp}) ; 68.1$ (d, $\left.\mathrm{J}_{\mathrm{PC}}=9.9 \mathrm{~Hz}: \mathrm{CpCH}_{2}\right) ; 22.0\left(\mathrm{~s}: \mathrm{CH}_{3}\right) .{ }^{31} \mathrm{P}$ NMR $\left(81.0 \mathrm{MHz}, \mathrm{CDCl}_{3}\right), \delta$ (ppm): -22.9 $\left(\mathrm{PPh}_{2}\right)$. HR MS (DCI $\left.\mathrm{CH}_{4}\right), \mathrm{C}_{31} \mathrm{H}_{29} \mathrm{OPFe}$, calcd. mass [M+H]: 505.1375; exp. mass $[\mathrm{M}+\mathrm{H}]: 505.1384$.

\subsubsection{Synthesis and characterization of 4-(bromomethyl)benzyl alcohol}

At $0^{\circ} \mathrm{C}$, in a Schlenk tube, $65 \mathrm{~mL}$ of $\mathrm{BH}_{3}$.THF (1M in THF, $65 \mathrm{mmol}$ ) were added to $11.6 \mathrm{~g}$ of 4-(bromomethyl)benzoic acid (54 mmol) in $20 \mathrm{~mL}$ of freshly distilled THF, under argon. After $2 \mathrm{~h}$ stirring at room temperature, $20 \mathrm{~mL}$ of methanol were added slowly. The clear solution was concentrated. The crude product was solubilized in ether and washed with an aqueous saturated solution of $\mathrm{NaHCO}_{3}$. After concentration, $10.4 \mathrm{~g}$ of a white solid were obtained (yield = 96\%) without further purification. ${ }^{1} \mathrm{H}$ NMR $\left(200.1 \mathrm{MHz}, \mathrm{CDCl}_{3}\right), \delta$ (ppm): 7.46-7.30 (4H, m: Ar); $4.73\left(2 \mathrm{H}, \mathrm{s}: \mathrm{CH}_{2}-\mathrm{Br}\right) ; 4.54\left(2 \mathrm{H}, \mathrm{s}: \mathrm{CH}_{2}-\mathrm{OH}\right) .{ }^{13} \mathrm{C} \mathrm{NMR}$ (50.3 MHz, $\left.\mathrm{CDCl}_{3}\right), \delta$ (ppm): 142.0 (s: quat Ar); 138.0 (s: quat Ar); 130.0 (s: Ar); 128.1 (s: Ar); 65.66 (s: $\left.\mathrm{CH}_{2} \mathrm{OH}\right) ; 34.01$ (s: $\mathrm{CH}_{2} \mathrm{Br}$ ). Anal. Calcd. for $\mathrm{C}_{8} \mathrm{H}_{9} \mathrm{BrO}$ : C, $47.79 \%$; $\mathrm{H}, 4.51 \%$. Found: C, 47.85\%; H, $4.53 \%$.

\subsubsection{Synthesis and characterization of compound 3}

In a Schlenk tube, $1.5 \mathrm{~g}$ of 2-thiodiphenylphosphino(hydroxymethyl)ferrocene 1 (3.47 mmol) was dissolved in $15 \mathrm{~mL}$ of dry dichloromethane. Then $1.5 \mathrm{~mL}$ of a $54 \%$ solution of tetrafluoroboric acid in ether $(10.86 \mathrm{mmol})$ was added. After $1 \mathrm{~min}$ stirring, a solution of $5.12 \mathrm{~g}$ of 4-(bromomethyl)benzylalcohol in $12 \mathrm{~mL}$ of dry dichloromethane was added. After $1 \mathrm{~min}$ of stirring, the crude material was filtered on silica gel with ether as an eluent. After evaporation of the solvent, $1.5 \mathrm{~g}$ of $\mathbf{3}$ was obtained as a yellow solid (yield =70\%). ${ }^{1} \mathrm{H}$ NMR $(300.1 \mathrm{MHz}$, $\left.\mathrm{CDCl}_{3}\right), \delta(\mathrm{ppm}): 7.87-7.80\left(2 \mathrm{H}, \mathrm{m}: \mathrm{PPh}_{2}\right) ; 7.72-7.65$ (2H, m: $\left.\mathrm{PPh}_{2}\right) ; 7.55-7.40\left(4 \mathrm{H}, \mathrm{m}: \mathrm{PPh}_{2}\right)$; 7.39-7.30 (2H, m: $\left.\mathrm{PPh}_{2}\right) ; 7.23(2 \mathrm{H}, \mathrm{d}, \mathrm{J}=8.0 \mathrm{~Hz}: \mathrm{Ph}) ; 6.98(2 \mathrm{H}, \mathrm{d}, \mathrm{J}=8.0 \mathrm{~Hz}: \mathrm{Ph}) ; 5.01(1 \mathrm{H}$, d, J = $\left.10.8 \mathrm{~Hz}: \mathrm{CpCH}_{2}\right)$; 4.65 (1H, m: subst Cp); $4.48\left(2 \mathrm{H}, \mathrm{s}: \mathrm{CH}_{2} \mathrm{Br}\right) ; 4.44$ (1H, d, J = 10.8 Hz: $\left.\mathrm{CpCH}_{2}\right) ; 4.34$ (5H, s: Cp); $4.34\left(2 \mathrm{H}, \mathrm{s}: \mathrm{PhCH}_{2} \mathrm{O}\right) ; 4.30$ (1H, m: subst Cp); 3.84 (1H, m: subst Cp). ${ }^{13} \mathrm{C}$ NMR (75.5 MHz, $\mathrm{CDCl}_{3}$ ), $\delta$ (ppm): 138.9 (s: quat Ph); 136.6 (s: quat Ph); 134.9 (d, $\mathrm{J}_{\mathrm{PC}}=87.4 \mathrm{~Hz}:$ quat $\left.\mathrm{PPh}_{2}\right) ; 133.6\left(\mathrm{~d}, \mathrm{~J}_{\mathrm{PC}}=86.5 \mathrm{~Hz}:\right.$ quat $\left.\mathrm{PPh}_{2}\right) ; 132.2\left(\mathrm{~d}, \mathrm{~J}_{\mathrm{PC}}=10.0 \mathrm{~Hz}: \mathrm{PPh}_{2}\right)$; $132.0\left(\mathrm{~d}, \mathrm{~J}_{\mathrm{PC}}=10.0 \mathrm{~Hz}: \mathrm{PPh}_{2}\right) ; 131.3\left(\mathrm{~d}, \mathrm{~J}_{\mathrm{PC}}=2.5 \mathrm{~Hz}: \mathrm{PPh}_{2}\right) ; 131.1\left(\mathrm{~d}, \mathrm{~J}_{\mathrm{PC}}=2.5 \mathrm{~Hz}: \mathrm{PPh}_{2}\right)$; 128.9 (s: Ph); 127.9 (s: Ph); 128.1 (d, JPC $=12.5$ Hz: $\mathrm{PPh}_{2}$ ); 128.0 (d, JPC $=12.5$ Hz: $\mathrm{PPh}_{2}$ ); 88.0 $\left(\mathrm{d}, \mathrm{J}_{\mathrm{PC}}=12.0 \mathrm{~Hz}\right.$ : quat Cp); $75.3\left(\mathrm{~d}, \mathrm{~J}_{\mathrm{PC}}=12.6 \mathrm{~Hz}\right.$ : subst Cp); 74.7 (d, $\mathrm{J}_{\mathrm{PC}}=94.9 \mathrm{~Hz}$ : quat Cp); $74.6\left(\mathrm{~d}, \mathrm{~J}_{\mathrm{PC}}=9.3 \mathrm{~Hz}\right.$ : subst Cp); $72.0\left(\mathrm{~s}: \mathrm{PhCH}_{2} \mathrm{O}\right.$ ); 70.7 (s: Cp); 69.5 (d, J $\mathrm{JC}_{\mathrm{PC}}=10.0 \mathrm{~Hz}$ : subst 
Cp); 67.1 (s: $\mathrm{CpCH}_{2} \mathrm{O}$ ); 33.6 (s: $\mathrm{CH}_{2} \mathrm{Br}$ ). ${ }^{31} \mathrm{P} \mathrm{NMR}$ (121.5 MHz, $\mathrm{CDCl}_{3}$ ), $\delta$ (ppm): 41.8. MS (FAB >0; MNBA), $\mathrm{C}_{31} \mathrm{H}_{28} \mathrm{BrFeOPS}, \mathrm{m} / \mathrm{z}: 616\left([\mathrm{M}]^{+}\right)$.

\subsubsection{Synthesis and characterization of compounds $\mathbf{4 a}$ and $\mathbf{4 c}$.}

In a Schlenk tube, $250 \mathrm{mg}$ of tetraethylmethylenebisphosphonate $(0.87 \mathrm{mmol})$ were reacted with a suspension of $250 \mathrm{mg}$ of sodium hydride (60\% dispersion in mineral oil) in $5 \mathrm{~mL}$ of THF. After $1 \mathrm{~h}$ at room temperature, the filtered solution was transferred via cannula on 500 $\mathrm{mg}$ of compound $3(0.81 \mathrm{mmol})$ dissolved in $20 \mathrm{~mL}$ of THF. After 1 day, $10 \mathrm{~mL}$ of a saturated aqueous solution of ammonium chloride were added and the product was extracted with ethyl acetate. The crude material was purified by flash chromatography on silica gel with ethyl acetate/ethanol (9/1) as eluent. Compounds 3, 4a and 4c were successively recovered. After evaporation of the solvent, $270 \mathrm{mg}$ of $\mathbf{4 a}$ was obtained as an orange solid (yield $=40 \%$ ). ${ }^{1} \mathrm{H}$ NMR (200.1 MHz, $\left.\mathrm{CDCl}_{3}\right), \delta(\mathrm{ppm}): 7.87-7.62$ (4H, m: $\left.\mathrm{PPh}_{2}\right) ; 7.50-7.16\left(6 \mathrm{H}, \mathrm{m}: \mathrm{PPh}_{2}\right) ; 7.14$ $(2 \mathrm{H}, \mathrm{d}, \mathrm{J}=8.1 \mathrm{~Hz}: \mathrm{Ph}) ; 6.96(2 \mathrm{H}, \mathrm{d}, \mathrm{J}=8.1 \mathrm{~Hz}: \mathrm{Ph}) ; 4.38\left(1 \mathrm{H}, \mathrm{d}, \mathrm{J}=11.0 \mathrm{~Hz}: \mathrm{CpCH}_{2}\right) .4 .89$ $\left(1 \mathrm{H}, \mathrm{dd}, \mathrm{J}=11.0 \mathrm{~Hz}: \mathrm{CpCH}_{2}\right) ; 4.63(1 \mathrm{H}, \mathrm{m}$ : subst $\mathrm{Cp}) ; 4.31\left(2 \mathrm{H}, \mathrm{s}: \mathrm{PhCH}_{2} \mathrm{O}\right) ; 4.31$ (5H, s: Cp); 4.27 (1H, m: subst Cp); 4.20-4.00 (8H, m: $\mathrm{CH}_{2}$ phosphonates); 3.82 (1H, m: subst $\left.\mathrm{Cp}\right) ; 3.21$ $\left(2 \mathrm{H}, \mathrm{t}\right.$ of $\left.\mathrm{d}, \mathrm{J}=6.0 \mathrm{~Hz}, \mathrm{~J}_{\mathrm{PH}}=16.7 \mathrm{~Hz}: \mathrm{PhCH}_{2}\right) ; 2.62\left(1 \mathrm{H}, \mathrm{t}\right.$ of $\mathrm{t}, \mathrm{J}=6.2 \mathrm{~Hz}, \mathrm{~J}_{\mathrm{PH}}=23.9 \mathrm{~Hz}$ : $\left.\mathrm{CHP}_{2}\right) ; 1.28\left(12 \mathrm{H}, \mathrm{t}\right.$ of $\mathrm{d}, \mathrm{J}=7.1 \mathrm{~Hz}, \mathrm{~J}_{\mathrm{PH}}=2.6 \mathrm{~Hz}: \mathrm{CH}_{3}$ phosphonates $) .{ }^{13} \mathrm{C} \mathrm{NMR}(50.3 \mathrm{MHz}$, $\left.\mathrm{CDCl}_{3}\right), \delta(\mathrm{ppm}): 135.7\left(\mathrm{~d}, \mathrm{~J}_{\mathrm{PC}}=87.6 \mathrm{~Hz}:\right.$ quat $\left.\mathrm{PPh}_{2}\right) ; 139.4\left(\mathrm{dd}, \mathrm{J}_{\mathrm{PC}}=7.3 \mathrm{~Hz}:\right.$ quat $\left.\mathrm{Ph}\right) ; 134.3$ $\left(\mathrm{d}, \mathrm{J}_{\mathrm{PC}}=86.3 \mathrm{~Hz}:\right.$ quat $\left.\mathrm{PPh}_{2}\right) ; 137.5(\mathrm{~s}:$ quat $\mathrm{Ph}) ; 132.9\left(\mathrm{~d}, \mathrm{~J}_{\mathrm{PC}}=10.1 \mathrm{~Hz}: \mathrm{PPh}_{2}\right) ; 132.8\left(\mathrm{~d}, \mathrm{~J}_{\mathrm{PC}}=\right.$ $\left.10.1 \mathrm{~Hz}: \mathrm{PPh}_{2}\right) ; 132.0$ (d, JPC $\left.=2.4 \mathrm{~Hz}: \mathrm{PPh}_{2}\right) ; 131.8\left(\mathrm{~d}, \mathrm{~J}_{\mathrm{PC}}=2.4 \mathrm{~Hz}: \mathrm{PPh}_{2}\right) ; 129.4$ (s: Ph); $128.9\left(\mathrm{~d}, \mathrm{~J}_{\mathrm{PC}}=12.8 \mathrm{~Hz}: \mathrm{PPh}_{2}\right) ; 128.8\left(\mathrm{~d}, \mathrm{~J}_{\mathrm{PC}}=12.4 \mathrm{~Hz}: \mathrm{PPh}_{2}\right) ; 128.3(\mathrm{~s}: \mathrm{Ph}) ; 89.0\left(\mathrm{~d}, \mathrm{~J}_{\mathrm{PC}}=11.9\right.$ Hz: quat Cp); 75.0 (d, JPC $=11.8 \mathrm{~Hz}$ : subst Cp); 75.3 (d, $\mathrm{J}_{\mathrm{PC}}=95.3 \mathrm{~Hz}$ : quat Cp); 75.2 (d, $\mathrm{J}_{\mathrm{PC}}=$ 9.3 Hz: subst Cp); 72.0 (s: $\mathrm{PhCH}_{2} \mathrm{O}$ ); 71.47 (s: Cp); 70.2 (d, $\mathrm{J}_{\mathrm{PC}}=10.4 \mathrm{~Hz}$ : subst Cp); 67.5 (s: $\left.\mathrm{CpCH}_{2} \mathrm{O}\right) ; 63.4\left(\mathrm{~d}, \mathrm{~J}_{\mathrm{PC}}=7.4 \mathrm{~Hz}: \mathrm{CH}_{2}\right.$ phosphonates); 63.2 (d, $\mathrm{J}_{\mathrm{PC}}=7.7 \mathrm{~Hz}: \mathrm{CH}_{2}$ phosphonates); $39.8\left(\mathrm{t}, \mathrm{J}_{\mathrm{PC}}=132.5 \mathrm{~Hz}: \mathrm{CHP}_{2}\right) ; 31.6\left(\mathrm{t}, \mathrm{J}_{\mathrm{PC}}=4.3 \mathrm{~Hz}: \mathrm{PhCH}_{2}\right) ; 17.1\left(\mathrm{~d}, \mathrm{~J}_{\mathrm{PC}}=6.4\right.$ $\mathrm{Hz}$ : $\mathrm{CH}_{3}$ phosphonates). ${ }^{31} \mathrm{P} \mathrm{NMR}\left(81.0 \mathrm{MHz} \mathrm{CDCl}_{3}\right), \delta$ (ppm): 24.3 (phosphonates); 43.0 $\left(\mathrm{PPh}_{2}\right)$. MS (FAB >0; MNBA), $\mathrm{C}_{40} \mathrm{H}_{49} \mathrm{FeO}_{7} \mathrm{P}_{3} \mathrm{~S}, \mathrm{~m} / \mathrm{z}: 845\left([\mathrm{M}+\mathrm{Na}]^{+}\right), 822\left([\mathrm{M}]^{+}\right)$.

After evaporation of the solvent, $60 \mathrm{mg}$ of $\mathbf{4 c}$ were recovered as a waxy solid. ${ }^{1} \mathrm{H}$ NMR (200.1 MHz, $\left.\mathrm{CDCl}_{3}\right), \delta(\mathrm{ppm}): 7.93-7.68\left(8 \mathrm{H}, \mathrm{m}: \mathrm{PPh}_{2}\right) ; 7.56-7.33\left(12 \mathrm{H}, \mathrm{m}: \mathrm{PPh}_{2} ; 4 \mathrm{H}, \mathrm{d}, \mathrm{J}=\right.$ $7.8 \mathrm{~Hz}: \mathrm{Ph}) ; 7.02$ (4H, d, J = 7.8 Hz: Ph); $4.91\left(2 \mathrm{H}, \mathrm{d}, \mathrm{J}=11.0 \mathrm{~Hz}: \mathrm{CpCH}_{2}\right) ; 4.69$ (2H, m: subst $\mathrm{Cp}) ; 4.50\left(2 \mathrm{H}, \mathrm{d}, \mathrm{J}=11.0 \mathrm{~Hz}: \mathrm{CpCH}_{2}\right) ; 4.37-4.34\left(4 \mathrm{H}, \mathrm{s}: \mathrm{PhCH}_{2} \mathrm{O} ; 10 \mathrm{H}\right.$, s: Cp; $2 \mathrm{H}$, m: subst $\mathrm{Cp})$; 4.00-4.10 (8H, m: $\mathrm{CH}_{2}$ phosphonates); $3.88(2 \mathrm{H}, \mathrm{m}$ : subst $\mathrm{Cp}), 3.34\left(4 \mathrm{H}, \mathrm{dd}, \mathrm{J}_{\mathrm{PH}}=16.1\right.$ 
Hz: $\left.\mathrm{PhCH}_{2}\right) ; 1.19\left(12 \mathrm{H}\right.$, dd, J = 7.0 Hz: $\mathrm{CH}_{3}$ phosphonates). ${ }^{13} \mathrm{C} \mathrm{NMR}\left(50.3 \mathrm{MHz}, \mathrm{CDCl}_{3}\right), \delta$ (ppm): $136.7(\mathrm{~s}:$ quat $\mathrm{Ph}) ; 135.6\left(\mathrm{dd}, \mathrm{J}_{\mathrm{PC}}=6.1 \mathrm{~Hz}:\right.$ quat $\left.\mathrm{Ph}\right) ; 134.9\left(\mathrm{~d}, \mathrm{~J}_{\mathrm{PC}}=87.8 \mathrm{~Hz}\right.$ : quat $\left.\mathrm{PPh}_{2}\right) ; 133.6\left(\mathrm{~d}, \mathrm{~J}_{\mathrm{PC}}=85.7 \mathrm{~Hz}:\right.$ quat $\left.\mathrm{PPh}_{2}\right) ; 132.2\left(\mathrm{~d}, \mathrm{~J}_{\mathrm{PC}}=10.0 \mathrm{~Hz}: \mathrm{PPh}_{2}\right) ; 132.0\left(\mathrm{~d}, \mathrm{~J}_{\mathrm{PC}}=10.1\right.$ Hz: $\left.\mathrm{PPh}_{2}\right) ; 131.5$ (s: Ph); 131.3 (d, JPC $\left.=2.2 \mathrm{~Hz}: \mathrm{PPh}_{2}\right) ; 131.1$ (d, JPC $\left.=2.2 \mathrm{~Hz}: \mathrm{PPh}_{2}\right) ; 128.1(\mathrm{~d}$, $\left.\mathrm{J}_{\mathrm{PC}}=12.7 \mathrm{~Hz}: \mathrm{PPh}_{2}\right) ; 128.0\left(\mathrm{~d}, \mathrm{~J}_{\mathrm{PC}}=12.2 \mathrm{~Hz}: \mathrm{PPh}_{2}\right) ; 126.6(\mathrm{~s}: \mathrm{Ph}) ; 88.3\left(\mathrm{~d}, \mathrm{~J}_{\mathrm{PC}}=12.1 \mathrm{~Hz}:\right.$ quat Cp); $75.2\left(\mathrm{~d}, \mathrm{~J}_{\mathrm{PC}}=12.3 \mathrm{~Hz}\right.$ : subst Cp); 75.5 (d, JPC = 94.8 Hz: quat Cp); 74.4 (d, JPC = 9.4 Hz: subst Cp); 72.3 (s, $\mathrm{PhCH}_{2} \mathrm{O}$ ); 70.7 (s: Cp); 69.4 (d, $\mathrm{J}_{\mathrm{PC}}=10.3 \mathrm{~Hz}$ : subst $\mathrm{Cp}$ ); 66.7 (s: $\mathrm{CpCH}_{2} \mathrm{O}$ ); 62.3 (d, $\mathrm{J}_{\mathrm{PC}}=4.6 \mathrm{~Hz}: \mathrm{CH}_{2}$ phosphonates); 48.7 (t, $\left.\mathrm{J}_{\mathrm{PC}}=132.7 \mathrm{~Hz}: \mathrm{CP}_{2}\right) ; 37.3$ (d, J $\mathrm{J}_{\mathrm{PC}}=4.2 \mathrm{~Hz}$ : $\left.\mathrm{PhCH}_{2}\right) ; 16.2\left(\mathrm{~d}, \mathrm{~J}_{\mathrm{PC}}=4.9 \mathrm{~Hz}: \mathrm{CH}_{3}\right.$ phosphonates). ${ }^{31} \mathrm{P} \mathrm{NMR}\left(81.0 \mathrm{MHz}, \mathrm{CDCl}_{3}\right), \delta(\mathrm{ppm})$ : $43.1\left(\mathrm{PPh}_{2}\right) ; 25.7$ (phosphonates). $\mathrm{MS}$ (FAB >0; MNBA), $\mathrm{C}_{71} \mathrm{H}_{76} \mathrm{Fe}_{2} \mathrm{O}_{8} \mathrm{P}_{4} \mathrm{~S}_{2}, \mathrm{~m} / \mathrm{z}: 1379$ $\left([\mathrm{M}+\mathrm{Na}]^{+}\right), 1356\left([\mathrm{M}]^{+}\right)$.

\subsubsection{Synthesis and characterization of compound $\mathbf{4 b}$}

In a Schlenk tube, $180 \mathrm{mg}$ of $\mathbf{4 a}(0.22 \mathrm{mmol})$ were dissolved in $5 \mathrm{~mL}$ of dry toluene with 0.24 $\mathrm{mL}$ of tris(dimethylamino)phosphine $(1.3 \mathrm{mmol})$. The solution was kept at reflux overnight under argon. After cooling back to room temperature, the solution was evaporated in vacuo. The crude residue was purified under argon by flash chromatography on silica gel with dichloromethane as eluent. After solvent evaporation, $120 \mathrm{mg}$ of $\mathbf{4 b}$ were obtained as an orange waxy solid (yield = 70\%). ${ }^{1} \mathrm{H}$ NMR $\left(300.1 \mathrm{MHz}, \mathrm{CDCl}_{3}\right), \delta$ (ppm): 7.60-7.50 (2H, m: $\mathrm{PPh}_{2}$ ); 7.40-7.38 (3H, m: $\left.\mathrm{PPh}_{2}\right)$; 7.25- 7.23 (5H, m: $\left.\mathrm{PPh}_{2}\right)$; 7.13 (2H, d, J = 8.1 Hz: Ph); 6.95 (2H, d, J $=8.1 \mathrm{~Hz}: \mathrm{Ph}) ; 4.58\left(1 \mathrm{H}, \mathrm{dd}, \mathrm{J}=10.8 \mathrm{~Hz}, \mathrm{~J}_{\mathrm{PH}}=2.2 \mathrm{~Hz}: \mathrm{CpCH}_{2}\right) ; 4.54(1 \mathrm{H}, \mathrm{m}:$ subst Cp$) ; 4.38$ $\left(1 \mathrm{H}, \mathrm{d}, \mathrm{J}=10.8 \mathrm{~Hz}: \mathrm{CpCH}_{2}\right)$; 4.32 (2H, s: $\left.\mathrm{PhCH}_{2} \mathrm{O}\right)$; 4.31 (1H, m: subst $\left.\mathrm{Cp}\right) ; 4.11$ (8H, quint., $\mathrm{J}_{\mathrm{HH}}=\mathrm{J}_{\mathrm{PH}}=6.9 \mathrm{~Hz}: \mathrm{CH}_{2}$ phosphonates); $4.02(5 \mathrm{H}, \mathrm{s}: \mathrm{Cp}) ; 3.79(1 \mathrm{H}, \mathrm{m}:$ subst $\mathrm{Cp}) ; 3.21$ (2H, t of $\left.\mathrm{d}, \mathrm{J}=16.3 \mathrm{~Hz}, \mathrm{~J}_{\mathrm{PH}}=6.2 \mathrm{~Hz}: \mathrm{PhCH}_{2}\right) ; 2.62\left(1 \mathrm{H}, \mathrm{t}\right.$ of $\left.\mathrm{t}, \mathrm{J}=6.2 \mathrm{~Hz}, \mathrm{~J}_{\mathrm{PH}}=24.0 \mathrm{~Hz}: \mathrm{CHP}_{2}\right) ; 1.28$ $\left(12 \mathrm{H}, \mathrm{t}\right.$ of $\mathrm{d}, \mathrm{J}=6.9 \mathrm{~Hz}, \mathrm{~J}_{\mathrm{PH}}=4.9 \mathrm{~Hz}: \mathrm{CH}_{3}$ phosphonates). ${ }^{13} \mathrm{C} \mathrm{NMR}\left(75.5 \mathrm{MHz}, \mathrm{CDCl}_{3}\right), \delta$ (ppm): $140.0\left(\mathrm{~d}, \mathrm{~J}_{\mathrm{PC}}=9.6 \mathrm{~Hz}:\right.$ quat $\left.\mathrm{PPh}_{2}\right) ; 138.6\left(\mathrm{t}, \mathrm{J}_{\mathrm{PC}}=7.1 \mathrm{~Hz}:\right.$ quat $\left.\mathrm{Ph}\right) ; 137.5\left(\mathrm{~d}, \mathrm{~J}_{\mathrm{PC}}=8.4\right.$ Hz: quat $\left.\mathrm{PPh}_{2}\right) ; 136.7$ (s: quat $\left.\mathrm{Ph}\right) ; 135.1$ (d, JPC $\left.=21.3 \mathrm{~Hz}: \mathrm{PPh}_{2}\right) ; 132.4\left(\mathrm{~d}, \mathrm{~J}_{\mathrm{PC}}=18.1 \mathrm{~Hz}\right.$ : $\left.\mathrm{PPh}_{2}\right) ; 129.2\left(\mathrm{~s}: \mathrm{PPh}_{2}\right) ; 128.7$ (s: Ph); 128.1 (d, JPC $\left.=8 \mathrm{~Hz}: \mathrm{PPh}_{2}\right) ; 128.0\left(\mathrm{~d}, \mathrm{~J}_{\mathrm{PC}}=6.2 \mathrm{~Hz}\right.$ : $\left.\mathrm{PPh}_{2}\right) ; 127.7\left(\mathrm{~s}: \mathrm{PPh}_{2}\right) ; 127.4(\mathrm{~s}: \mathrm{Ph}) ; 89.2$ (d, JPC = 24.6 Hz: quat Cp); 76.6 (d, JPC = 8.0 Hz: quat $\mathrm{Cp}) ; 72.6\left(\mathrm{~d}, \mathrm{~J}_{\mathrm{PC}}=3.5 \mathrm{~Hz}\right.$ : subst $\left.\mathrm{Cp}\right) ; 72.0\left(\mathrm{~s}, \mathrm{PhCH}_{2} \mathrm{O}\right) ; 71.9$ (d, $\mathrm{J}_{\mathrm{PC}}=3 \mathrm{~Hz}$ : subst Cp); 69.9 (s: subst Cp); 69.6 (s: Cp); 67.4 (d, JPC $\left.=10.0 \mathrm{~Hz}: \mathrm{CpCH}_{2}\right) ; 62.6$ (d, JPC $=6.7 \mathrm{~Hz}: \mathrm{CH}_{2}$ phosphonates); 62.5 (d, JPC $=6.7 \mathrm{~Hz}: \mathrm{CH}_{2}$ phosphonates); 39.1 (t, $\mathrm{J}_{\mathrm{PC}}=132.1 \mathrm{~Hz}: \mathrm{CHP}_{2}$ ); 30.9 $\left(\mathrm{t}, \mathrm{J}_{\mathrm{PC}}=4.3 \mathrm{~Hz}: \mathrm{PhCH}_{2}\right) ; 16.3\left(\mathrm{~d}, \mathrm{~J}_{\mathrm{PC}}=6.3 \mathrm{~Hz}: \mathrm{CH}_{3}\right.$ phosphonates $) ;{ }^{31} \mathrm{P} \mathrm{NMR}(121.5 \mathrm{MHz}$, 
$\left.\mathrm{CDCl}_{3}\right), \delta$ (ppm): 23.1 (phosphonates); -22.1 $\left(\mathrm{PPh}_{2}\right)$. MS (FAB >0; MNBA), $\mathrm{C}_{40} \mathrm{H}_{49} \mathrm{FeO}_{7} \mathrm{P}_{3}$, $\mathrm{m} / \mathrm{z}: 829\left([\mathrm{M}+\mathrm{K}]^{+}\right), 790\left([\mathrm{M}]^{+}\right)$.

\subsubsection{Synthesis and characterization of compound $\mathbf{5 a}$}

In a schlenk tube, $100 \mathrm{mg}$ of $\mathbf{3}$ were reacted with $100 \mu \mathrm{L} \mathrm{N}$-methylimidazole in $15 \mathrm{~mL}$ of refluxing toluene. After eight hours, the solution was cooled down to room temperature. The precipitate was filtered off and washed with toluene then ether and dried in vacuo. The product 5a was obtained as a yellow solid (yield $=90 \mathrm{mg}, 80 \%) .{ }^{1} \mathrm{H}$ NMR (200.1 MHz, MeOD), $\delta$ (ppm): 7.89-7.77 (2H, m: $\mathrm{PPh}_{2}$ ); 7.69-7.35 (8H, m: $\mathrm{PPh}_{2} ; 2 \mathrm{H}:$ imidazolium); 7.28 (2H, d, J = $8.1 \mathrm{~Hz}: \mathrm{Ph}) ; 7.05(2 \mathrm{H}, \mathrm{d}, \mathrm{J}=8.1 \mathrm{~Hz}: \mathrm{Ph}) ; 5.39\left(2 \mathrm{H}, \mathrm{s}: \mathrm{PhCH}_{2} \mathrm{~N}\right) ; 5.07(1 \mathrm{H}, \mathrm{d}, \mathrm{J}=10.8 \mathrm{~Hz}$ : $\left.\mathrm{CpCH}_{2}\right)$; 4.72 (1H, m: subst Cp); 4.48 (1H, m: subst Cp); 4.39 (1H, d, J = 10.8 Hz: $\left.\mathrm{CpCH}_{2}\right)$; $4.38\left(2 \mathrm{H}\right.$, s: $\left.\mathrm{PhCH}_{2} \mathrm{O}\right)$; 4.33 (5H, s: $\left.\mathrm{Cp}\right) ; 3.96$ (3H, s: $\mathrm{CH}_{3}$ imidazolium); 3.89 (1H, m: subst $\mathrm{Cp})$. Imidazolium $\mathrm{NC} \underline{\mathrm{HN}}$ signal is observed in $\mathrm{CDCl}_{3}$ at $10.8(1 \mathrm{H}, \mathrm{s}) .{ }^{13} \mathrm{C} \mathrm{NMR}(50.3 \mathrm{MHz}$, $\mathrm{CDCl}_{3}$ ), $\delta$ (ppm): 140.49 (s: quat Ph); 133.4 (s: quat Ph); 138.0 (s: NCHN imidazolium); 134.2 $\left(\mathrm{d}, \mathrm{J}_{\mathrm{PC}}=84.9 \mathrm{~Hz}:\right.$ quat $\left.\mathrm{PPh}_{2}\right) ; 133.6\left(\mathrm{~d}, \mathrm{~J}_{\mathrm{PC}}=86.5 \mathrm{~Hz}:\right.$ quat $\left.\mathrm{PPh}_{2}\right) ; 132.7\left(\mathrm{~d}, \mathrm{~J}_{\mathrm{PC}}=8.0 \mathrm{~Hz}\right.$ : $\left.\mathrm{PPh}_{2}\right) ; 132.5\left(\mathrm{~d}, \mathrm{~J}_{\mathrm{PC}}=8.1 \mathrm{~Hz}: \mathrm{PPh}_{2}\right) ; 132.0\left(\mathrm{~d}, \mathrm{~J}_{\mathrm{PC}}=2.6 \mathrm{~Hz}: \mathrm{PPh}_{2}\right) ; 131.7\left(\mathrm{~d}, \mathrm{~J}_{\mathrm{PC}}=2.6 \mathrm{~Hz}\right.$ : $\mathrm{PPh}_{2}$ ); 129.0 (s: Ph); 128.9 (s: Ph); 128.7 (d, JPC = 4.2 Hz: PPh $) ; 128.4$ (d, JPC = 4.2 Hz: PPh $)_{\text {; }}$ 124.4 (s: CH imidazolium); 122.9 (s: CH imidazolium); 88.0 (d, J $\mathrm{J}_{\mathrm{PC}}=12.0 \mathrm{~Hz}$ : quat Cp); 76.0 $\left(\mathrm{d}, \mathrm{J}_{\mathrm{PC}}=12.0 \mathrm{~Hz}\right.$ : subst Cp); $75.6\left(\mathrm{~d}, \mathrm{~J}_{\mathrm{PC}}=9.1 \mathrm{~Hz}\right.$ : subst Cp); $74.7\left(\mathrm{~d}, \mathrm{~J}_{\mathrm{PC}}=92.0 \mathrm{~Hz}:\right.$ quat Cp$)$; 72.0 (s, $\left.\mathrm{PhCH}_{2} \mathrm{O}\right) ; 71.1$ (s: Cp); 70.3 (d, $\mathrm{J}_{\mathrm{PC}}=10.3 \mathrm{~Hz}$ : subst Cp); 67.4 (s: $\left.\mathrm{CpCH}_{2} \mathrm{O}\right) ; 53.2$ (s: $\mathrm{PhCH}_{2} \mathrm{~N}$ ); 35.4 (s: $\mathrm{CH}_{3}$ imidazolium). ${ }^{31} \mathrm{P}$ NMR (81.0 MHz, MeOD), $\delta$ (ppm): 43.0. MS (FAB $>0$; MNBA), $\mathrm{C}_{35} \mathrm{H}_{34} \mathrm{FeN}_{2} \mathrm{OPS}, \mathrm{m} / \mathrm{z}: 617\left([\mathrm{M}]^{+}\right)$.

\subsubsection{Synthesis and characterization of compound $\mathbf{5 b}$}

In a Schlenk tube, $38 \mathrm{mg}$ of $\mathbf{5 a}(3.47 \mathrm{mmol})$ were reacted with $2 \mathrm{~g}$ of Raney nickel powder (W.R. Grace and Co. Raney ${ }^{\circledR} 2800$, slurry, in $\mathrm{H}_{2} \mathrm{O}$ ) in $6 \mathrm{~mL}$ of acetonitrile at $50^{\circ} \mathrm{C}$. Twenty four hours later the solution was filtered. After evaporation of the solvent, $\mathbf{5 b}$ was obtained as a yellow powder. ${ }^{1} \mathrm{H}$ NMR (200.1 $\left.\mathrm{MHz}, \mathrm{CDCl}_{3}\right), \delta$ (ppm): 10.7 (1H, s: NCHN imidazolium); 7.61-7.52 (2H, m: $\mathrm{PPh}_{2}$; 1H: imidazolium); 7.43-7.37 (4H, m: $\left.\mathrm{PPh}_{2}\right)$; 7.25-7.22 (4H, m: $\mathrm{PPh}_{2}$; 2H: Ph); 7.11 (1H, br s: imidazolium); $6.99(2 \mathrm{H}, \mathrm{d}, \mathrm{J}=8.0 \mathrm{~Hz}: \mathrm{Ph}) .5 .5\left(2 \mathrm{H}, \mathrm{s}: \mathrm{PhCH}_{2} \mathrm{~N}\right) ; 4.68$ $\left(1 \mathrm{H}, \mathrm{dd}, \mathrm{J}=10.8 \mathrm{~Hz}, \mathrm{~J}_{\mathrm{PH}}=2.2 \mathrm{~Hz}: \mathrm{CpCH}_{2}\right) ; 4.56(1 \mathrm{H}, \mathrm{m}$ : subst Cp); $4.41(1 \mathrm{H}, \mathrm{d}, \mathrm{J}=10.8 \mathrm{~Hz}$ : $\left.\mathrm{CpCH}_{2}\right) ; 4.34\left(2 \mathrm{H}, \mathrm{s}: \mathrm{PhCH}_{2} \mathrm{O}\right) ; 4.33$ (1H, m: subst $\left.\mathrm{Cp}\right) ; 4.06$ (3H, s: $\mathrm{CH}_{3}$ imidazolium); 4.03 (5H, s: Cp); 3.81 (1H, m: subst Cp). ${ }^{13} \mathrm{C} \mathrm{NMR} \mathrm{(50.3} \mathrm{MHz,} \mathrm{CDCl}_{3}$ ), $\delta$ (ppm): 140.3 (s: quat Ph); 
$140.1\left(\mathrm{~d}, \mathrm{~J}_{\mathrm{PC}}=9.5 \mathrm{~Hz}:\right.$ quat $\left.\mathrm{PPh}_{2}\right) ; 138.0$ (s: NCHN imidazolium); 137.3 (d, JPC $=7.4 \mathrm{~Hz}$ : quat $\left.\mathrm{PPh}_{2}\right) ; 135.1\left(\mathrm{~d}, \mathrm{~J}_{\mathrm{PC}}=21.1 \mathrm{~Hz}: \mathrm{PPh}_{2}\right) ; 132.4\left(\mathrm{~d}, \mathrm{~J}_{\mathrm{PC}}=17.9 \mathrm{~Hz}: \mathrm{PPh}_{2}\right) ; 131.3(\mathrm{~s}:$ quat Ph$) ; 129.3$ $\left(\mathrm{s}: \mathrm{PPh}_{2}\right) ; 129.0$ (s: Ph); 128.5 (s: Ph); 128.2 (d, JPC $\left.=7.2 \mathrm{~Hz}: \mathrm{PPh}_{2}\right) ; 128.1\left(\mathrm{~d}, \mathrm{~J}_{\mathrm{PC}}=5.3 \mathrm{~Hz}\right.$ : $\left.\mathrm{PPh}_{2}\right) ; 127.9\left(\mathrm{~s}: \mathrm{PPh}_{2}\right) ; 123.0\left(\mathrm{~s}: \mathrm{CH}\right.$ imidazolium); 121.5 (s: $\mathrm{CH}$ imidazolium); 88.7 (d, $\mathrm{J}_{\mathrm{PC}}=$ 25.5 Hz: quat Cp); 72.9 (d, $\mathrm{J}_{\mathrm{PC}}=2.9 \mathrm{~Hz}$ : subst $\mathrm{Cp}$ ); $72.2\left(\mathrm{~d}, \mathrm{~J}_{\mathrm{PC}}=3.0 \mathrm{~Hz}\right.$ : subst Cp); 71.5 (s, $\mathrm{PhCH}_{2} \mathrm{O}$ ); 70.1 (s: subst Cp); 69.6 (s: Cp); 67.9 (d, J $\left.\mathrm{JC}_{\mathrm{PC}}=9.7 \mathrm{~Hz}: \mathrm{CpCH}_{2}\right) ; 53.4\left(\mathrm{~s}: \mathrm{CH}_{2} \mathrm{~N}\right) ; 36.8$ (s: $\mathrm{CH}_{3}$ imidazolium). ${ }^{31} \mathrm{P}$ NMR (81.0 MHz, $\left.\mathrm{CDCl}_{3}\right), \delta$ (ppm): -22.4.

\subsubsection{Synthesis and characterization of compound $\boldsymbol{6} \boldsymbol{a}$}

In a Schlenk tube, $720 \mathrm{mg}$ of monomethylether mPEG 750 were reacted with a suspension of $210 \mathrm{mg}$ of sodium hydride $(60 \%$ dispersion in mineral oil) in $6 \mathrm{~mL}$ of THF. After $1 \mathrm{~h}$ at room temperature, the solution was transferred via cannula on $90 \mathrm{mg}$ of $\mathbf{3}$ dissolved in $4 \mathrm{~mL}$ of THF. Twenty-four hours later, $10 \mathrm{~mL}$ of a saturated aqueous solution of ammonium chloride were added and the product was extracted with ethyl acetate and washed with water saturated with $\mathrm{NaCl}$. After solvent evaporation, $110 \mathrm{mg}$ of $\mathbf{6 a}$ were obtained as a yellow wax (yield $=60 \%$ ). ${ }^{1} \mathrm{H}$ NMR (200.1 MHz, $\left.\mathrm{CDCl}_{3}\right), \delta$ (ppm): 7.87-7.63 (4H, m: $\left.\mathrm{PPh}_{2}\right) ; 7.55-7.30$ (6H, m: $\mathrm{PPh}_{2}$ ); $7.18(2 \mathrm{H}, \mathrm{d}, \mathrm{J}=8.0 \mathrm{~Hz}: \mathrm{Ph}) ; 6.99(2 \mathrm{H}, \mathrm{d}, \mathrm{J}=8.0 \mathrm{~Hz}: \mathrm{Ph}) ; 4.92\left(1 \mathrm{H}, \mathrm{d}, \mathrm{J}=10.8 \mathrm{~Hz}: \mathrm{CpCH}_{2}\right)$; 4.64 (1H, m: subst Cp); $4.52\left(2 \mathrm{H}\right.$, s: $\left.\mathrm{PhCH}_{2} \mathrm{O}\right) ; 4.42\left(1 \mathrm{H}, \mathrm{d}, \mathrm{J}=10.8 \mathrm{~Hz}: \mathrm{CpCH}_{2}\right)$; 4.31 (5H, s: Cp); 4.29 (2H, s: $\left.\mathrm{PhCH}_{2} \mathrm{O}\right)$; 4.29 (1H, m: subst Cp); 3.81 (1H, m: subst Cp); 3.75-3.55 (64H, m, $\mathrm{CH}_{2}-\mathrm{PEG}$ ); 3.38 (3H, s, $\left.\mathrm{CH}_{3} \mathrm{O}-\mathrm{PEG}\right) .{ }^{13} \mathrm{C} \mathrm{NMR}\left(50.3 \mathrm{MHz}, \mathrm{CDCl}_{3}\right.$ ), $\delta$ (ppm): 137.8 (s: quat $\mathrm{Ph}) ; 137.1$ (s: quat $\mathrm{Ph}) ; 134.8\left(\mathrm{~d}, \mathrm{~J}_{\mathrm{PC}}=87.3 \mathrm{~Hz}\right.$ : quat $\left.\mathrm{PPh}_{2}\right) ; 133.6\left(\mathrm{~d}, \mathrm{~J}_{\mathrm{PC}}=85.5 \mathrm{~Hz}\right.$ : quat $\left.\mathrm{PPh}_{2}\right) ; 132.1$ (d, JPC $=10.9$ Hz: $\left.\mathrm{PPh}_{2}\right) ; 132.0$ (d, JPC $\left.=10.9 \mathrm{~Hz}: \mathrm{PPh}_{2}\right) ; 131.2$ (d, JPC = $2.5 \mathrm{~Hz}:$ $\left.\mathrm{PPh}_{2}\right) ; 131.1\left(\mathrm{~d}, \mathrm{~J}_{\mathrm{PC}}=2.5 \mathrm{~Hz}: \mathrm{PPh}_{2}\right) ; 128.1\left(\mathrm{~d}, \mathrm{~J}_{\mathrm{PC}}=12.4 \mathrm{~Hz}: \mathrm{PPh}_{2}\right) ; 128.0\left(\mathrm{~d}, \mathrm{~J}_{\mathrm{PC}}=12.4 \mathrm{~Hz}\right.$ : $\left.\mathrm{PPh}_{2}\right) ; 127.8$ (2 C, s: Ph); 88.1 (d, JPC = 12.0 Hz: quat Cp); 75.2 (d, J $\mathrm{JC}_{\mathrm{PC}}=12.6 \mathrm{~Hz}$ : subst Cp);

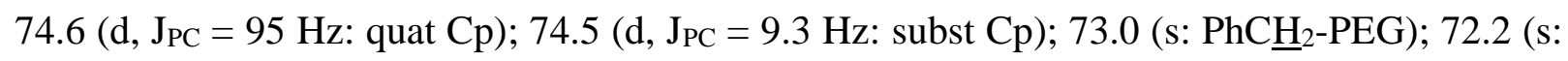
$\mathrm{CH}_{2}$ ); 71.9 (s: $\mathrm{CH}_{2}$ ); 70.5 (s: Cp); 70.5 (s: $\mathrm{CH}_{2}-\mathrm{PEG}$ ); 69.4 (d, JPC = 10.1 Hz: subst Cp); 69.2 (s: $\mathrm{CH}_{2}-\mathrm{PEG}$ ); 66.8 (s: $\mathrm{CH}_{2}$ ); 59.1 (s: $\left.\mathrm{CH}_{3} \mathrm{O}-\mathrm{PEG}\right) .{ }^{31} \mathrm{P}$ NMR (81.0 MHz, $\mathrm{CDCl}_{3}$ ), $\delta$ (ppm): 43.0.

\subsubsection{Synthesis and characterization of compound $\mathbf{6} \boldsymbol{b}$}

In a Schlenk tube, $80 \mathrm{mg}$ of $\mathbf{6 a}$ were dissolved in $2 \mathrm{~mL}$ of dry toluene with $0.40 \mathrm{~mL}$ of tris(dimethylamino)phosphine. The solution was kept at reflux overnight under argon. After cooling back to room temperature, the solution was evaporated in vacuo. The crude residue was 
purified under argon by flash chromatography on silica gel with dichloromethane as eluent. After evaporation of the solvent, $35 \mathrm{mg}$ of $\mathbf{6 b}$ were obtained as an orange waxy solid (yield = 45\%). ${ }^{1} \mathrm{H}$ NMR (200.1 MHz, $\left.\mathrm{CDCl}_{3}\right), \delta(\mathrm{ppm}): 7.62-7.52\left(2 \mathrm{H}, \mathrm{m}: \mathrm{PPh}_{2}\right) ; 7.41-7.36$ (3H, m: $\mathrm{PPh}_{2}$ ); 7.25-7.23 (5H, m: $\left.\mathrm{PPh}_{2}\right) ; 7.18(2 \mathrm{H}, \mathrm{d}, \mathrm{J}=8.1 \mathrm{~Hz}: \mathrm{Ph}) ; 6.98$ (2H, d, J = 8.1 Hz: Ph). 4.60 $\left(1 \mathrm{H}, \mathrm{dd}, \mathrm{J}=10.9 \mathrm{~Hz}, \mathrm{~J}=2.3 \mathrm{~Hz}: \mathrm{CpCH}_{2}\right) ; 4.55(1 \mathrm{H}, \mathrm{m}:$ subst Cp$) ; 4.52\left(2 \mathrm{H}, \mathrm{s}: \mathrm{PhCH}_{2} \mathrm{O}\right) ; 4.38$ $\left(1 \mathrm{H}, \mathrm{d}, \mathrm{J}=10.9 \mathrm{~Hz}: \mathrm{CpCH}_{2}\right) ; 4.33\left(2 \mathrm{H}, \mathrm{s}: \mathrm{PhCH}_{2} \mathrm{O}\right) ; 4.31$ (1H, m: subst Cp); 4.02 (5H, s: Cp); 3.78 (1H, m: subst Cp); 3.66-3.65 (64H, m, $\left.\mathrm{CH}_{2}-\mathrm{PEG}\right) ; 3.39$ (3H, s, $\left.\mathrm{CH}_{3} \mathrm{O}-\mathrm{PEG}\right) .{ }^{13} \mathrm{C}$ NMR (50.3 MHz, $\left.\mathrm{CDCl}_{3}\right), \delta(\mathrm{ppm}): 139.9\left(\mathrm{~d}, \mathrm{~J}_{\mathrm{PC}}=8.9 \mathrm{~Hz}:\right.$ quat $\left.\mathrm{PPh}_{2}\right) ; 137.8$ (s: quat $\left.\mathrm{Ph}\right) ; 137.4(\mathrm{~d}$, $\mathrm{J}_{\mathrm{PC}}=8.2 \mathrm{~Hz}:$ quat $\left.\mathrm{PPh}_{2}\right) ; 137.1(\mathrm{~s}:$ quat $\mathrm{Ph}) ; 135.1\left(\mathrm{~d}, \mathrm{~J}_{\mathrm{PC}}=20.6 \mathrm{~Hz}: \mathrm{PPh}_{2}\right) ; 132.4\left(\mathrm{~d}, \mathrm{~J}_{\mathrm{PC}}=17.9\right.$ Hz: $\left.P_{P h}\right) ; 129.1\left(\mathrm{~s}: \mathrm{PPh}_{2}\right) ; 128.1\left(\mathrm{~d}, \mathrm{~J}_{\mathrm{PC}}=7.4 \mathrm{~Hz}: \mathrm{PPh}_{2}\right) ; 128.0\left(\mathrm{~d}, \mathrm{~J}_{\mathrm{PC}}=5.5 \mathrm{~Hz}: \mathrm{PPh}_{2}\right) ; 127.7$ $\left(\mathrm{s}: \mathrm{PPh}_{2}\right) ; 127.7$ (s: Ph); 127.5 (s: Ph); 89.1 (d, $\mathrm{J}_{\mathrm{PC}}=25.0 \mathrm{~Hz}:$ quat $\left.\mathrm{Cp}\right) ; 76.7$ (d, $\mathrm{J}_{\mathrm{PC}}=8.0 \mathrm{~Hz}$ : quat Cp); 73.1 (s: $\left.\mathrm{PhC} \underline{\mathrm{H}}_{2}-\mathrm{PEG}\right) ; 72.8\left(\mathrm{~d}, \mathrm{~J}_{\mathrm{PC}}=3.6 \mathrm{~Hz}\right.$ : subst Cp); $72.0\left(\mathrm{~d}, \mathrm{~J}_{\mathrm{PC}}=3.7 \mathrm{~Hz}\right.$ : subst Cp); 72.0 (s: $\mathrm{CH}_{2}$ ); 70.9 (s: $\mathrm{CH}_{2}-\mathrm{PEG}$ ); 69.8 (s: subst Cp); 69.5 (s: Cp); 67.5 (s, J $\mathrm{JP}_{\mathrm{PC}}=9.7 \mathrm{~Hz}$ : $\mathrm{CpCH}_{2}$ ); 59.1 (s: $\left.\mathrm{CH}_{3} \mathrm{O}-\mathrm{PEG}\right) .{ }^{31} \mathrm{P} \mathrm{NMR}\left(81.0 \mathrm{MHz}, \mathrm{CDCl}_{3}\right), \delta$ (ppm): -22.0.

\subsection{General procedure for coupling reaction of aryl bromides with boronic acids}

In a typical procedure for coupling reaction (Scheme 2), ligand $\mathbf{2 b}$ (6 mg, $0.012 \mathrm{mmol}, 1.2 \mathrm{~mol}$ $\%), \mathrm{Pd}_{2}(\mathrm{dba})_{3}(5 \mathrm{mg}, 0.005 \mathrm{mmol}, 1.1 \mathrm{~mol} \%)$, phenylboronic acid (146 mg, $\left.1.2 \mathrm{mmol}\right)$ and cesium carbonate $(750 \mathrm{mg}, 2.030 \mathrm{mmol}, 2.3 \mathrm{~mol} \%$ ) were introduced into a Schlenk tube under an argon stream. A solution of bromobenzene $(157 \mathrm{mg}, 1.00 \mathrm{mmol})$ in $10 \mathrm{~mL}$ of dioxane was added into the Schlenk tube via a syringe. The reaction mixture was stirred at $70^{\circ} \mathrm{C}$ for $8 \mathrm{~h}$ under an $\mathrm{Ar}$ atmosphere. Aliquots $(0.5 \mathrm{~mL})$ of the solution were taken using a polypropylene syringe. One $\mathrm{mL}$ of water was added in each collected samples. After extraction by two portions of $1 \mathrm{~mL}$ of diethylether, the organic phase was then filtered on silica and analysed by GPC using diethylene glycol dibutyl ether as internal standard.

\subsection{X-ray diffraction studies.}

A single crystal of compound $\mathbf{3}$ was mounted under inert perfluoropolyether at the tip of a glass fibre and cooled in the cryostream of a Bruker APEXII CCD diffractometer. Data were collected using the monochromatic MoK $\alpha$ radiation $(\lambda=0.71073)$. The structure was solved by direct methods (SIR97 [41]) and refined by least-squares procedures on $\mathrm{F}^{2}$ using SHELXL-97 [42]. All $\mathrm{H}$ atoms attached to carbon were introduced in calculation in idealised positions and treated as riding models. There is half of a dichloromethane solvate per asymmetric unit located around a 
twofold axis. The drawing of the molecules was realized with the help of ORTEP32 [43]. Crystal data and refinement parameters are shown in Table 1. Crystallographic data (excluding structure factors) have been deposited with the Cambridge Crystallographic Data Centre as supplementary publication number CCDC 745960. Copies of the data can be obtained free of charge on application to the Director, CCDC, 12 Union Road, Cambridge CB2 1EZ, UK (fax: (+44) 1223336-033; e-mail: deposit@ccdc.cam.ac.uk).

\section{Acknowledgement}

The authors thank LCC CNRS for financial support, the Chemistry Department of "IUT A Paul Sabatier" for financial support and for offering accesses to some analytical equipment, and L. Orts and T. Scandella for laboratory assistance. 


\section{Figure captions}

Table $1-{ }^{31} \mathrm{P}$ and $\left.{ }^{13} \mathrm{C}\left(\underline{\mathrm{C}} \mathrm{H}_{2} \mathrm{R}\right)\right) \mathrm{NMR}$ data $\left(\mathrm{CDCl}_{3}, \delta\right.$ in ppm).

Table 2 - Crystal data and structure refinement parameters for compound $\mathbf{3}$.

Table 3 - Bond distances $(\AA)$ and bond angles $\left(^{\circ}\right)$ with e.s.d.s in parentheses for the structure of compound 3 .

Figure 1 - Suzuki-Miyaura reaction of $\mathrm{PhBr}$ with $\mathrm{PhB}(\mathrm{OH})_{2}$ in the presence of $\mathrm{Pd}_{2}(\mathrm{dba})_{3}$ with and without ligand 2 (Reaction conditions: dioxane, $70^{\circ} \mathrm{C}, \mathrm{PhBr} / \mathrm{Pd} /$ ligand $/ \mathrm{Cs}_{2} \mathrm{CO}_{3}=$ 100:1:1.2:2.3).

Figure 2 - An ORTEP view of the structure of compound 3. Ellipsoids are drawn at the $30 \%$ probability level. The dichloromethane solvate and $\mathrm{H}$ atoms have been omitted for clarity.

Scheme 1 - Synthesis of tagged ligands.

Scheme 2 - Synthesis of ligand $\mathbf{2 b}$.

Scheme 3 - Reagents and conditions for Suzuki- Miyaura reaction.

Scheme 4 - Synthesis of compounds 4 to 6.

Scheme 5 - Synthesis of compounds $\mathbf{4 a}$ and $\mathbf{4 c}$. 
Table $1-{ }^{31} \mathrm{P}$ and ${ }^{13} \mathrm{C}\left(\underline{\mathrm{C}} \mathrm{H}_{2} \mathrm{R}\right.$ ') $\mathrm{NMR}$ data $\left(\delta\right.$ in $\left.\mathrm{CDCl}_{3}\right)$.

\begin{tabular}{|c|c|c|c|c|}
\hline compound & $R^{\prime}$ & ${ }^{31} \mathbf{P}\left\{{ }^{1} \mathrm{H}\right\}$ & \multicolumn{2}{|c|}{${ }^{13} \mathrm{C}\left\{{ }^{1} \mathrm{H}\right\}$} \\
\hline $2 a$ & $H$ & 43.1 & 21.3 & - \\
\hline $2 b$ & $H$ & -21.9 & 22.0 & - \\
\hline 3 & $B r$ & 41.8 & 33.6 & - \\
\hline $4 \mathbf{a}$ & $\mathrm{CH}\left[\mathrm{PO}(\mathrm{OEt})_{2}\right]_{2}$ & $\begin{array}{l}43.0 \\
24.3\end{array}$ & $\begin{array}{c}31.6 \\
\mathrm{dd}\end{array}$ & $\mathrm{J}_{\mathrm{PC}}=4.3 \mathrm{~Hz}$ \\
\hline $4 b$ & $\mathrm{CH}\left[\mathrm{PO}(\mathrm{OEt})_{2}\right]_{2}$ & $\begin{array}{c}23.1 \\
-22.1\end{array}$ & $\begin{array}{c}30.9 \\
\mathrm{dd}\end{array}$ & $\mathrm{J}_{\mathrm{PC}}=4.3 \mathrm{~Hz}$ \\
\hline $4 c$ & $\mathrm{C}\left[\mathrm{PO}(\mathrm{OEt})_{2}\right]_{2}$ & $\begin{array}{l}43.1 \\
25.7\end{array}$ & $\begin{array}{c}37.3 \\
\mathrm{dd}\end{array}$ & $\mathrm{J}_{\mathrm{PC}}=4.2 \mathrm{~Hz}$ \\
\hline $5 a$ & Imidazolium & 43.0 & 53.2 & - \\
\hline $5 b$ & Imidazolium & -22.4 & 53.4 & - \\
\hline $6 a$ & PEG 750 & 43.0 & 73.0 & - \\
\hline $6 \mathbf{b}$ & PEG 750 & -22.0 & 73.1 & - \\
\hline
\end{tabular}


Table 2 - Crystal data and structure refinement parameters for compound $\mathbf{3}$.

\begin{tabular}{|c|c|}
\hline Identification code & 3 \\
\hline Empirical formula & $\mathrm{C}_{31} \mathrm{H}_{28} \mathrm{BrFeOPS}, 1 / 2\left(\mathrm{CH}_{2} \mathrm{Cl}_{2}\right)$ \\
\hline Formula weight & 657.79 \\
\hline Temperature, $\mathrm{K}$ & $180(2)$ \\
\hline Crystal system & 0.71073 \\
\hline Space group & Monoclinic \\
\hline $\mathrm{a}, \AA$ & $C 2 / c$ \\
\hline $\mathrm{b}, \AA$ & $34.2054(8)$ \\
\hline c, $\AA$ & $9.1162(2)$ \\
\hline$\beta,^{\circ}$ & $114.370(3)$ \\
\hline $\mathrm{V}, \AA^{3}$ & $5643.5(2)$ \\
\hline $\mathrm{Z}$ & 8 \\
\hline Dcalc, $\mathrm{Mg} / \mathrm{m}^{3}$ & 1.548 \\
\hline$\mu, \mathrm{mm}^{-1}$ & 2.200 \\
\hline $\mathrm{F}(000)$ & 2680 \\
\hline Crystal size, $\mathrm{mm}^{3}$ & $0.31 \times 0.29 \times 0.03$ \\
\hline$\theta^{\circ}$, range & 2.08 to 29.07 \\
\hline Reflections collected & 69915 \\
\hline Unique reflections [ $\left.\mathrm{R}_{\text {int }}\right]$ & $7516[0.133]$ \\
\hline Obsd reflections $[\mathrm{I}>2 \sigma(\mathrm{I})]$ & 4731 \\
\hline Completeness $(\%)$ & 99.5 \\
\hline Absorption correction & Multi-scan \\
\hline $\mathrm{T}_{\min } / T_{\max }$ & 1.0 and 0.761 \\
\hline Refinement & $\mathrm{F}^{2}$ \\
\hline Parameters & 339 \\
\hline GOF on $F^{2}$ & 1.022 \\
\hline $\mathrm{R} 1, \mathrm{wR} 2[\mathrm{I}>2 \sigma(\mathrm{I})]$ & $0.0472,0.1173$ \\
\hline R1, wR2 (all data) & $0.0912,0.1339$ \\
\hline$\Delta \rho_{\min ,} \Delta \rho_{\max }\left(\mathrm{e} \AA^{-3}\right)$ & $1.008 /-1.123$ \\
\hline
\end{tabular}


Table 3 - Bond distances $(\AA)$ and bond angles $\left(^{\circ}\right)$ with e.s.d.s in parentheses for the structure of compound $\mathbf{3}$.

\begin{tabular}{|ll|ll|}
\hline $\mathrm{Br}(1)-\mathrm{C}(23)$ & $1.964(4)$ & $\mathrm{P}(1)-\mathrm{C}(111)$ & $1.816(3)$ \\
\hline $\mathrm{P}(1)-\mathrm{C}(1)$ & $1.790(3)$ & $\mathrm{P}(1)-\mathrm{S}(1)$ & $1.9623(11)$ \\
\hline $\mathrm{P}(1)-\mathrm{C}(121)$ & $1.808(3)$ & $\mathrm{O}(2)-\mathrm{C}(22)$ & $1.419(4)$ \\
\hline $\mathrm{O}(2)-\mathrm{C}(21)$ & $1.428(3)$ & $\mathrm{C}(22)-\mathrm{C}(221)$ & $1.493(4)$ \\
\hline $\mathrm{C}(2)-\mathrm{C}(21)$ & $1.488(4)$ & $\mathrm{C}(23)-\mathrm{C}(224)$ & $1.487(5)$ \\
\hline
\end{tabular}

\begin{tabular}{|ll|ll|}
\hline $\mathrm{C}(1)-\mathrm{P}(1)-\mathrm{C}(121)$ & $106.09(14)$ & $\mathrm{C}(1)-\mathrm{P}(1)-\mathrm{S}(1)$ & $116.62(10)$ \\
\hline $\mathrm{C}(1)-\mathrm{P}(1)-\mathrm{C}(111)$ & $104.45(13)$ & $\mathrm{C}(121)-\mathrm{P}(1)-\mathrm{S}(1)$ & $110.49(11)$ \\
\hline $\mathrm{C}(121)-\mathrm{P}(1)-\mathrm{C}(111)$ & $105.87(13)$ & $\mathrm{C}(111)-\mathrm{P}(1)-\mathrm{S}(1)$ & $112.53(10)$ \\
\hline $\mathrm{C}(22)-\mathrm{O}(2)-\mathrm{C}(21)$ & $111.6(2)$ & $\mathrm{O}(2)-\mathrm{C}(21)-\mathrm{C}(2)$ & $108.0(2)$ \\
\hline $\mathrm{C}(2)-\mathrm{C}(1)-\mathrm{P}(1)$ & $128.1(2)$ & $\mathrm{C}(5)-\mathrm{C}(1)-\mathrm{P}(1)$ & $123.8(2)$ \\
\hline $\mathrm{C}(3)-\mathrm{C}(2)-\mathrm{C}(21)$ & $125.8(3)$ & $\mathrm{C}(1)-\mathrm{C}(2)-\mathrm{C}(21)$ & $127.3(3)$ \\
\hline $\mathrm{O}(2)-\mathrm{C}(22)-\mathrm{C}(221)$ & $108.6(2)$ & $\mathrm{C}(224)-\mathrm{C}(23)-\mathrm{Br}(1)$ & $111.0(2)$ \\
\hline $\mathrm{C}(116)-\mathrm{C}(111)-\mathrm{P}(1)$ & $122.8(2)$ & $\mathrm{C}(126)-\mathrm{C}(121)-\mathrm{P}(1)$ & $122.7(2)$ \\
\hline $\mathrm{C}(112)-\mathrm{C}(111)-\mathrm{P}(1)$ & $118.2(2)$ & $\mathrm{C}(122)-\mathrm{C}(121)-\mathrm{P}(1)$ & $118.8(2)$ \\
\hline $\mathrm{C}(222)-\mathrm{C}(221)-\mathrm{C}(22)$ & $120.7(3)$ & $\mathrm{C}(226)-\mathrm{C}(221)-\mathrm{C}(22)$ & $121.2(3)$ \\
\hline $\mathrm{C}(223)-\mathrm{C}(224)-\mathrm{C}(23)$ & $120.5(3)$ & $\mathrm{C}(225)-\mathrm{C}(224)-\mathrm{C}(23)$ & $120.5(4)$ \\
\hline
\end{tabular}


Figure 1 - Suzuki-Miyaura reaction of $\mathrm{PhBr}$ with $\mathrm{PhB}(\mathrm{OH})_{2}$ in the presence of $\mathrm{Pd}_{2}(\mathrm{dba})_{3}$ with and without ligand 2 (Reaction conditions: dioxane, $70^{\circ} \mathrm{C}, \mathrm{PhBr} / \mathrm{Pd} /$ ligand $/ \mathrm{Cs}_{2} \mathrm{CO}_{3}=$ 100:1:1.2:2.3).

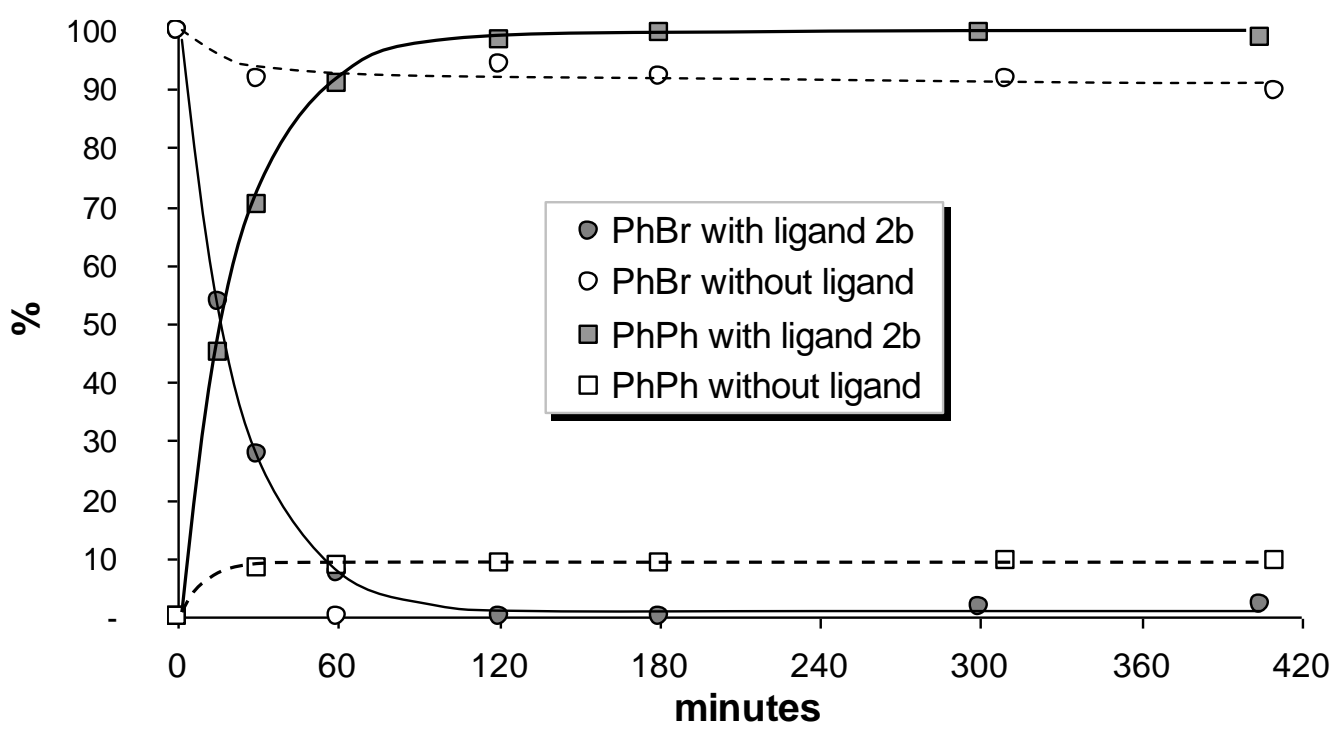


Figure 2 - An ORTEP view of the structure of compound 3. Ellipsoids are drawn at the $30 \%$ probability level. The dichloromethane solvate and $\mathrm{H}$ atoms have been omitted for clarity.

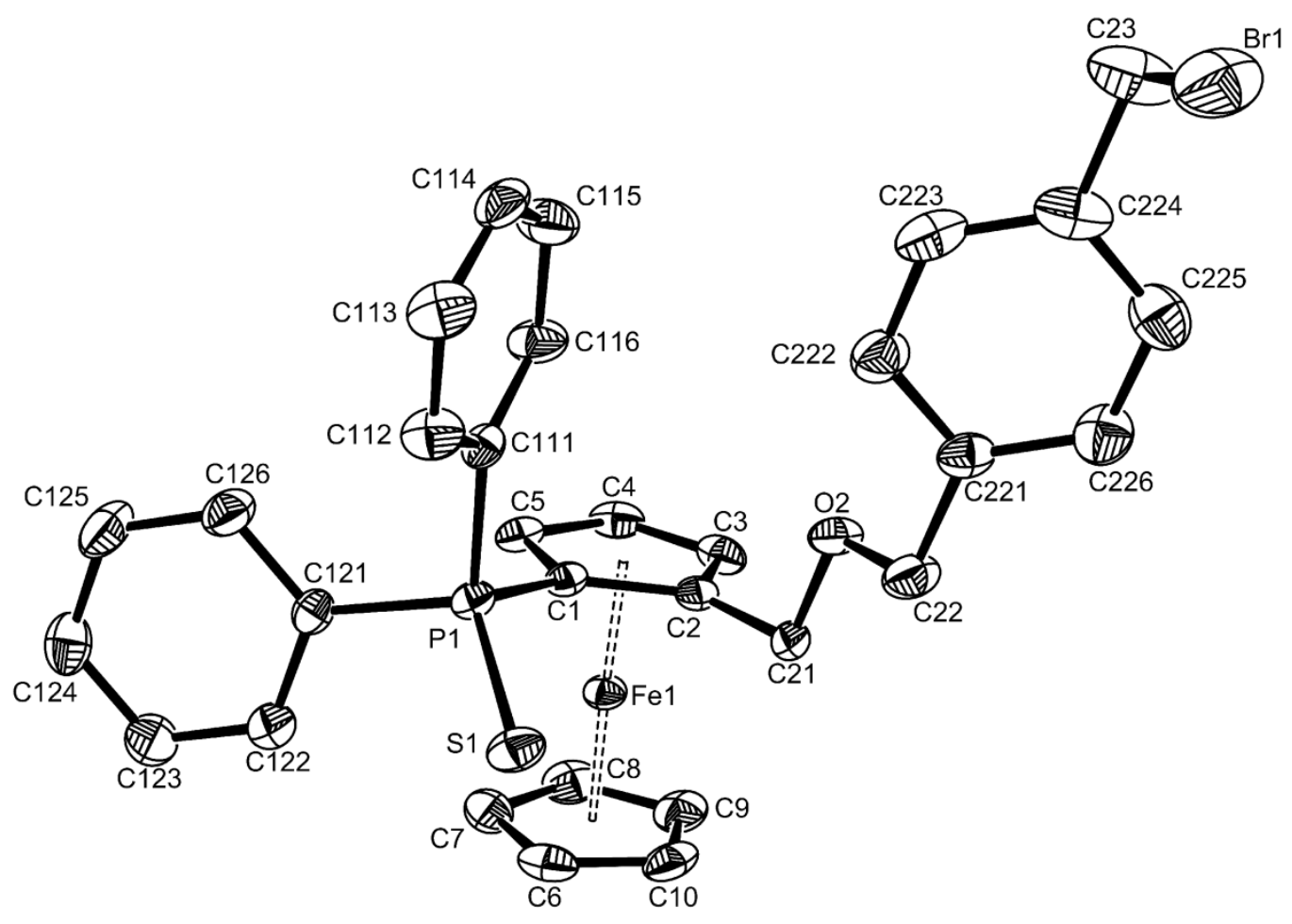


Scheme 1 - Synthesis of tagged-ligands.

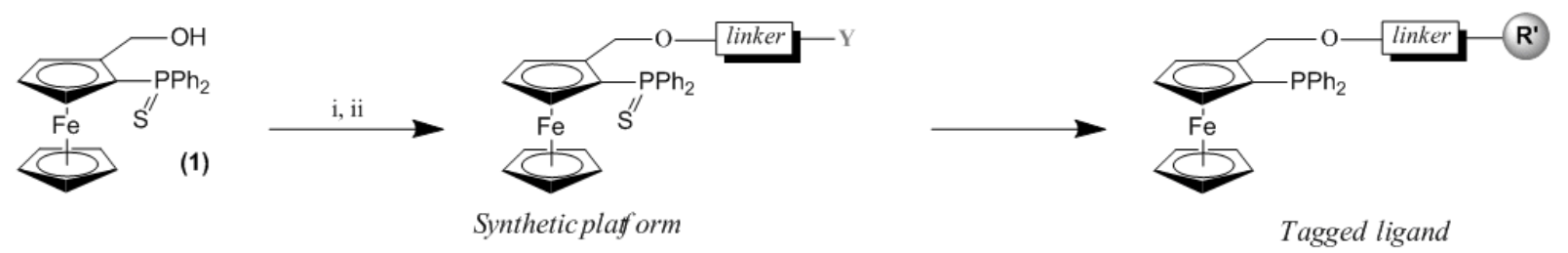

Conditions: (i) $\mathrm{HBF}_{4}$. (ii) HO-linker-Y 
Scheme 2 - Synthesis of ligand $\mathbf{2 b}$.
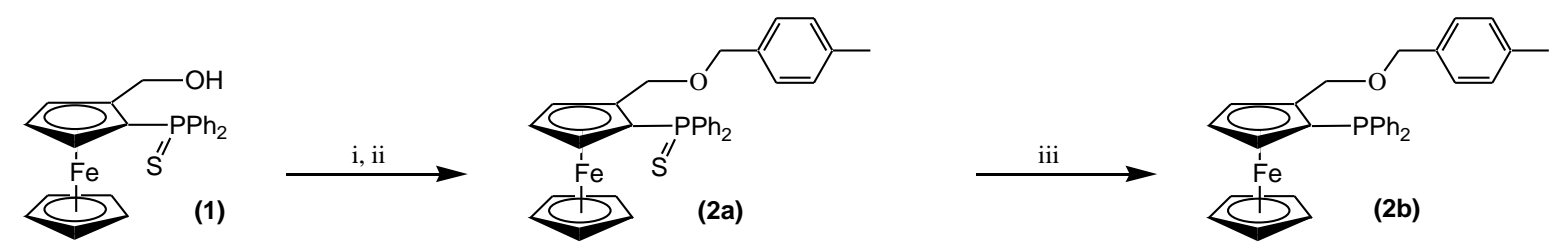

Conditions: (i) $\mathrm{HBF}_{4}$. (ii) $\mathrm{p}-\mathrm{HOCH}_{2}\left(\mathrm{C}_{6} \mathrm{H}_{4}\right) \mathrm{CH}_{3}$. (iii) $\mathrm{P}\left(\mathrm{NMe}_{2}\right)_{3}$, toluene reflux. 
Scheme 3 - Reagents and conditions for Suzuki- Miyaura reaction.

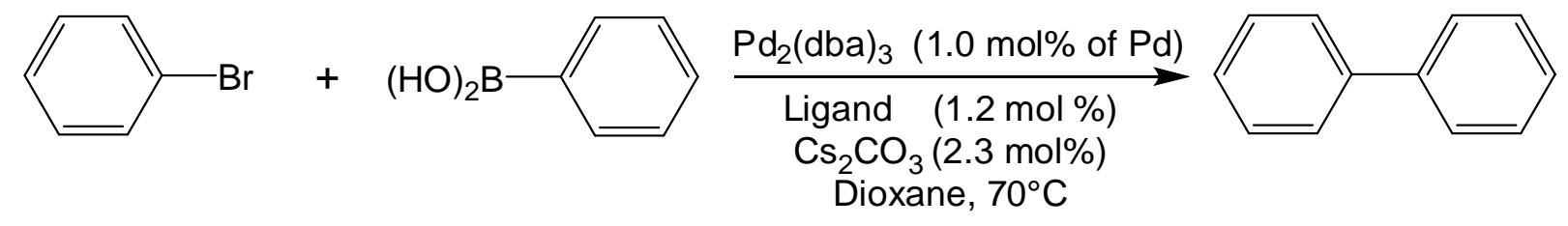


Scheme 4 - Synthesis of compound 4 to 6.

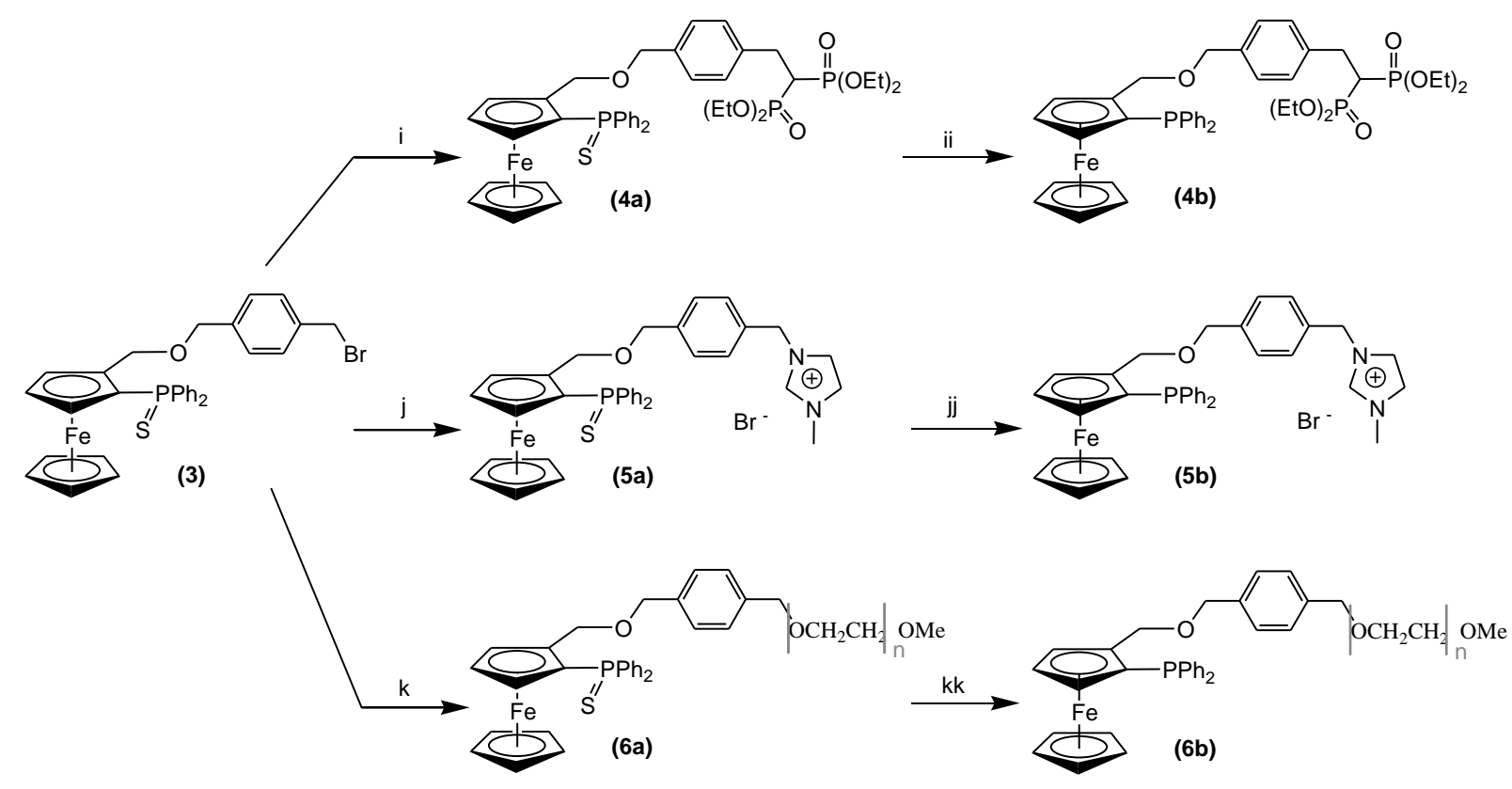

Conditions: (i) $\mathrm{NaH}$, tetraethylbisphosphonate. (ii) $\mathrm{P}\left(\mathrm{NMe}_{2}\right)_{3}$. (j) methylimidazole. (jj) Raney Ni. (k) NaH, monomethylether PEG 750. (kk) $\mathrm{P}\left(\mathrm{NMe}_{2}\right)_{3}$. 
Scheme 5 - Synthesis of compounds $\mathbf{4 a}$ and $\mathbf{4 c}$.

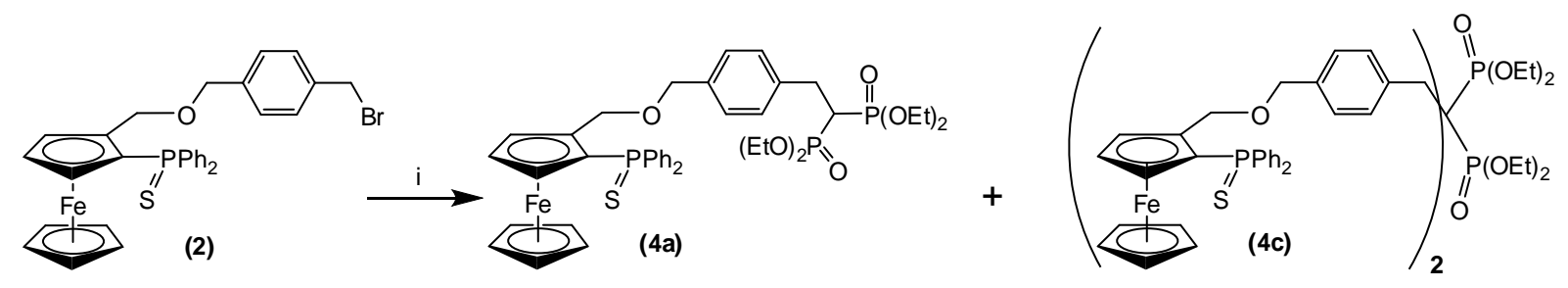




\section{References:}

1 (a) E. N. Jacobsen, A. Pfalz, H. Yamamoto Eds, Comprehensive Asymmetric Catalysis, Vols. 1-3, Springer, Berlin (1999). (b) A. Börner Ed., Phosphorus Ligands in Asymmetric Catalysis, Vols. 1-3, Wiley-VCH, Weinheim, Germany, (2008).

2 (a) A. N. Collins, G. N. Sheldrake, J. Crosby, Eds, Chirality in Industry: The Commercial Manufacture and Applications of Optically Active Compounds; Collins, John Wiley \& Sons: Chichester, (1992). (b) J. N. Hawkins, T. J. N. Watson, Angew. Chem. Int. Ed. 43 (2004) 3224-3228. (c) H.-U. Blaser, B. Pugin, F. Spindler, M. Thommen, Acc. Chem. Res. 40 (2007), 1240-1250

3 Q.-H. Fan, Y.-M. Li, A.S.C. Chan, Chem. Rev. 102 (2002) 3385-3466.

4 A. F. Trindade, P.M.P Gois, C. A. M. Alfonso, Chem. Rev. 109 (2009) 418-514.

$5 \quad$ P. Akiyama, S. Kobayashi, Chem. Rev. 109 (2009) 594-642.

6 J. Lu, P. H. Toy, Chem. Rev. 109 (2009) 815-838.

7 C. E. Song, S.-G. Lee, Chem. Rev. 102 (2002) 3495-3524.

8 (a) D. E. Bergbreiter, J. Tian, C. Hongfa, Chem. Rev. 109 (2009) 530-582. (b) T. J. Dickerson, N. N. Reed, K. D. Janda, Chem. Rev. 102 (2002) 3325-3344.

9 (a) L. J. Twyman, A. S. H. King, I. K. Martin, Chem. Soc. Rev. 31 (2002) 69-82. (b) R. van Heerbeek, P. C. J. Kamer, P. W. N. M. van Leeuwen, J. N. Reek, Chem. Rev. 102 (2002) 3717-3756. (c) O. Bourrier, A. K. Kakkar, J. Mater. Chem. 13 (2003) 1306-1315. (d) A. Dahan, M. Portnoy, J. Polym. Sci. Part A: Polym. Chem. 43 (2005) 235-262. (e) D. Méry, D. Astruc, Coord. Chem. Rev. 250 (2006) 1965-1979. (f) A. Berger, R. J. M. Klein Gebbink, Top. Organomet. Chem. (2006) 1-38. (g) A.-M. Caminade, P. Servin, R. Laurent, J.-P. Majoral, Chem. Soc. Rev. 37 (2008) 56-67. (h) E. De Jesus, J. C. Flores, Ind. Eng. Chem. Res. 47 (2008) 7968-7981.

10 S. Liu, J. Xiao, J. Mol. Catal. A: Chem. 270 (2007) 1-43.

11 P. Tundo, A. Perosa, Chem. Soc. Rev. 36 (2007) 532-550.

12 P. G. Jessop, T. Ikariya, R. Noyori, Chem. Rev. 99 (1999) 475-493.

13 L. P. Barthel-Rosa, J. A. Gladysz, Coord. Chem. Rev. 190-192 (1999) 587-605.

14 J. Durand, E. Teuma, M. Gomez, C. R. Chimie 10 (2007) 152-177.

15 H. Olivier-Bourbigou, L. Magna, J. Mol. Catal. A: Chemical 182-183 (2002) 419-437.

16 C.-J. Li, Chem. Rev. 105 (2005) 3095-3165.

17 K. H. Shaughnessy, Chem. Rev. 109 (2009) 643-710. 
18 J. Chen, S. K. Spear, J. G. Huddleston, R. D. Rogers, Green Chem. 7 (2005) 64-82.

19 E. Manoury, J. S. Fossey, H. Aït-Haddou, J.-C. Daran, G. G. A. Balavoine, Organometallics 19 (2000) 3736-3739.

20 S. Mourgues, D. Serra, F. Lamy, S. Vincendeau, J.-C. Daran, E. Manoury, M. Gouygou, Eur. J. Inorg. Chem. (2003) 2820-2826.

21 L. Routaboul, S. Vincendeau, J.-C. Daran, E. Manoury, Tetrahedron Asym. 16 (2005) 2685-2690.

22 J. G. Lopez Cortes, O. Ramon, S. Vincendeau, D. Serra, F Lamy, J.-C. Daran, E. Manoury, M. Gouygou, Eur. J. Inorg. Chem. (2006) 5148-5157.

23 N. Mateus, L. Routaboul, J.-C. Daran, E. Manoury, J. Organomet. Chem. 691 (2006) 2297-2310.

24 L. Routaboul, S. Vincendeau, C.-O.Turrin, A.-M. Caminade, J.-P. Majoral, J.-C. Daran, E. Manoury, J. Organomet. Chem. 692 (2007) 1064-1073.

25 E. Le Roux, R. Malacea, E. Manoury, R. Poli, L. Gonsalvi, M ; Peruzzini, Advanced Synthesis and Catalysis 349 (2007) 309-313.

26 A. Labande, J.-C. Daran, E. Manoury, R. Poli, Eur. J. Inorg. Chem. (2007) 1205-1209.

27 L. Diab, M. Gouygou, E. Manoury, P. Kalck, M. Urrutigoïty, Tet. Lett. 49 (2008) 51865189.

28 B. Breit, D. Breuniger, J. Am. Chem. Soc. 126 (2004) 10244-10245.

29 R. C. J. Atkinson, V. C. Gibson, N. J. Long, A. J. P. White, D. J. Williams, Organometallics 23 (2004) 2744-2752.

30 B. Breit, D. Breuniger, Eur. J. Org. Chem. (2005) 3916-3929.

31 S. Teo, Z. Wenig, T. S. A. Hor, Organometallics 25 (2006) 1199-1205.

32 H. W. Yu, Q. S. Tong, Y. R. Peng, L. Jia, J. C. Shi, Z. L. Jin, Chinese Chemical Letter 18 (2007) 37-40.

33 J. Yoshida, K. Itami, Chem. Rev. 102 (2002), 3693-3716.

34 (a) A. Corma, H. Garcia, A. Leyva, Tetrahedron, 60 (2004) 8553-8560. (b) A. Corma, H. Garcia, A. Leyva, Tetrahedron 61 (2005) 9848-9854. (c) M. Lombardo, M. Chiaruccci, C. Trombini, Green Chem. 11 (2009) 574. (d) R. Franzen, Y. Xu, Can. J. Chem. 83 (2005) 266-272. (e) O. Adidou, C. Goux-Henry, M. Safi, M. Soufiaoui, E. Framery, Tet. Lett. 49 (2008). (f) F. Fernandez, B. Cordzero, J. Durand, G. Muller, F. Malbosc, Y. Kihn, E. Teuma, M. Gomez, Dalton Trans. (2007) 5572-5581. (g) H. Hagiwara, K. H. Ko, T. Hoshi, T. Suzuki, Chem. Commun. (2007) 2838-2840. (h) M.-J. Jin, A. Taher, H.-J. Kang, M. Choi, R. Ryoo, Green Chem. (2009) 309-313. (i) N. Yan, X. Yang, Z. Fai, Y. Li, Y. Kou, P. J. Dyson, Organometallics 28 (2009) 937-939. 
35 (a) I. R. Butler, W. R. Cullen, F. G. Herring, N. R. Jagannathan, F. W. B. Einstein, R. Jones, Inorg. Chem. 25 (1986) 4534-4541. (b) T. Mizuta, Y. Imamura, K. Miyoshi, H. Yorimitsu, K. Oshima, Organometallics 24 (2005) 990-996. (c) P. Stepnicka, I. Cisarova, New J. Chem. 26 (2002) 1389-1396. (d) J.-C. Daran, E. Manoury, L. Routaboul, F. Rivals, Acta Cryst. Sect. C C62 (2006) m378-m380. (e) P. Stepnicka, I. Cisarova, J. Organomet. Chem. 691 (2006) 2863-2871. (f) D. Wechsler, M. A. Rankin, R. McDonald, M. J. Ferguson, G. Schatte, M. Stradiotto, Organometallics 26 (2007) 6418-6427.

36 A. M. Herring, B. D. Steffey, A. Miedaner, S. A. Wander, D. L. Dubois, Inorg. Chem. 34 (1995) 1100-1109.

37 S. A. Holstein, D. M. Cermak, D. F. Wiemer, K. Lewis, R. J. Hohl, Bioorg. \& Med. Chem. 6 (1998) 687-694.

38 D. Liu, W. Tang, X. Zhang,Org. Lett. 6 (2004) 513-516.

39 (a) G. Bringmann, A. J. Price Mortimer, P. A. Keller, M. J. Gresser, J. Garner, M. Breuning, Angew. Chem. Int. Ed. 44 (2005), 5384-5427. (b) O. Baudoin, Eur. J. Org. Chem. (2005) 4223-4229.

40 T. Hayashi, T. Mise, M. Fukushima, M. Kagotani, N. Nagashima, Y. Hamada, A. Matsumoto, S. Kawakami, M. Konishi, K. Yamamoto, M. Kumada, Bull. Chem. Soc. Jpn. 53 (1980) 1138-1151.

41 A. Altomare, M. C. Burla, M. Camalli, G. L. Cascarano, C. Giacovazzo, A. Guagliardi, A. G. G. Moliterni, G. Polidori, R. Spagna. SIR97 a program for automatic solution of crystal structures by direct methods. J. Appl. Cryst. 32 (1999) 115-119.

42 G. M. Sheldrick, Acta Cryst. (2008) A64, 112-122

43 L. J. Farrugia. ORTEP3 for Windows, J. Appl. Cryst. 30 (1997) 565. 